\title{
Simulating the vibrational quantum dynamics of molecules with photonics
}

\author{
Chris Sparrow ${ }^{1,2},{ }^{*}$ Enrique Martín-López ${ }^{3},{ }^{*}$ Nicola Maraviglia ${ }^{1}$, Alex Neville $^{1}$, \\ Christopher Harrold ${ }^{1}$, Jacques Carolan ${ }^{4}$, Yogesh N. Joglekar ${ }^{5}$, Toshikazu Hashimoto ${ }^{6}$, \\ Nobuyuki Matsuda ${ }^{7}$, Jeremy L. O'Brien ${ }^{1}$, David P. Tew ${ }^{8}$, and Anthony Laing ${ }^{1 \dagger}$ \\ ${ }^{1}$ Quantum Engineering and Technology Laboratories, \\ School of Physics and Department of Electrical and Electronic Engineering, University of Bristol, Bristol BS8 1TL, UK \\ ${ }^{2}$ Department of Physics, Imperial College London, SW' 2AZ, UK \\ ${ }^{3}$ Nokia Bell Labs, Broers Building, 21 J.J. Thomson Avenue, Cambridge, CB3 0FA, UK \\ ${ }^{4}$ Department of Electrical Engineering and Computer Science, \\ Massachusetts Institute of Technology, Cambridge, Massachusetts 02139, United States \\ ${ }^{5}$ Department of Physics, Indiana University Purdue University Indianapolis (IUPUI), Indianapolis, Indiana 46202 USA \\ ${ }^{6}$ NTT Device Technology Laboratories, NTT Corporation, \\ 3-1 Morinosato-Wakamiya, Atsugi, Kanagawa 243-0198, Japan \\ ${ }^{7}$ NTT Basic Research Laboratories, NTT Corporation, \\ 3-1 Morinosato-Wakamiya, Atsugi, Kanagawa 243-0198, Japan \\ ${ }^{8}$ School of Chemistry, University of Bristol, Bristol BSB 1TS, UK
}

\begin{abstract}
Advances in control techniques for vibrational quantum states in molecules present new challenges for modelling such systems that could be amenable to quantum simulation methods. Exploiting a natural mapping to photons in waveguides, we demonstrate a reprogrammable photonic chip as a versatile simulation platform for a range of quantum dynamical behaviour in different molecules. We begin by simulating the time evolution of vibrational excitations in the harmonic approximation for a variety of 4-atom molecules, including $\mathrm{H}_{2} \mathrm{CS}, \mathrm{SO}_{3}, \mathrm{HNCO}, \mathrm{HFHF}, \mathrm{N}_{4}$, and $\mathrm{P}_{4}$. We go on to simulate coherent and dephased energy transport in the simplest model of the peptide bond in proteins, N-methylacetamide, and simulate thermal relaxation and the effect of anharmonicities in $\mathrm{H}_{2} \mathrm{O}$. Finally, we use multi-photon statistics with a feedback control algorithm to iteratively identify quantum states that increase a particular dissociation pathway of $\mathrm{NH}_{3}$. These methods point to powerful new simulation tools for molecular quantum dynamics and the field of femtochemistry.
\end{abstract}

Early electronic computers exploited analogies with acoustic, thermal, or mechanical phenomena, such as capacitance for spring stiffness, to simulate a range of practically relevant physical systems $[1,2]$. While modern digital simulations have become versatile foundational tools in science and engineering, all classical computers are fundamentally inefficient at tackling exponentially complex microscopic behaviour such as the quantum dynamics of molecules $[3,4]$. A proposed solution is to engineer quantum mechanical components into devices that are then inherently capable of simulating quantum systems [5-10]. Here, we demonstrate how integrated quantum photonics can be used as a platform to develop new simulation methods for molecular quantum dynamics, by building on the analogies of optical modes in waveguides for vibrational modes in molecules and single photons for quantised vibrational excitations.

Advances in the control of ultrafast molecular dynamics have revealed the importance of quantum interference among vibrational modes in behaviour such as bond selective chemistry [4]. In applying optimal control theory to a harmonic model of chained atoms [11], it was shown in principle how a designed control field could drive the dynamics of quantum interference between vibrational modes [12] to excite local bonds. However, laboratory

\footnotetext{
* These authors contributed equally to this work.

$\dagger$ anthony.laing@bristol.ac.uk
}

demonstrations of selective bond dissociation required adaptive feedback control to put the principles into practice [13-16]. Further control over vibrational wavepackets has allowed selective dissociation governed by a single quantum of vibrational energy [17], manipulation of individual molecules at ambient conditions [18], preparations of coherent superpositions on sub-femtosecond timescales [19], and single vibrational states of ultracold molecules $[20,21]$. Molecular dynamics are now observable on their ultrafast intrinsic timescale $[22,23]$.

The prospect of more sophisticated control with quantum states of light and for larger molecules increases the challenge to simulate dynamical behaviour. Light-matter interaction with squeezed states has been experimentally demonstrated in a number of contexts [24-28] and enhanced spectroscopy and control of molecules with multimode, multi-photon states has been shown theoretically [29-31] with new techniques for pulse shaping of quantum states of light also demonstrated [32-35]. Evolving a multi-excitation state across many vibrational modes is computationally inefficient even for the basic model where normal modes are described as independent quantum harmonic oscillators. Due to their bosonic nature, the probability amplitudes for input-output transitions amongst the modes are determined by matrix permanents, the calculation of which is generally extremely complex [36, 37]. More detailed molecular models, for example with anharmonic corrections to the potentials, are also likely to be computationally complex.

This is the author's manuscript of the article published in final edited form as:

Sparrow, C., Martín-López, E., Maraviglia, N., Neville, A., Harrold, C., Carolan, J., ... Laing, A. (2018). Simulating the vibrational quantum dynamics of molecules using photonics. Nature, 557(7707), 660. https://doi.org/10.1038/s41586-018-0152-9 
Quantum algorithms for the efficient simulation of Hamiltonian dynamics $[6,38]$ have been a strong motivator for digital quantum computers, for example with trapped ions [39]. Promising digital algorithms for simulating reaction dynamics [40] and obtaining thermal rate constants [41] have been presented that harness the exponential quantum speed up. Yet, achieving fault tolerance [42] and the high logical gate counts [43] that enable these applications is extremely challenging. Ansatz based methods [44] for solving the eigenvalue problem have reduced demands, as recently demonstrated with superconducting qubits [45], but the difficulties associated with applying such an approach to Hamiltonian dynamics have yet to be overcome. Analogue quantum simulations [10], in which a quantum system of interest can be directly mapped onto a quantum technological platform, may enable practical advantages in the nearer term $[46,47]$.

Progress in photonic quantum technologies over the last decade has seen the introduction of on-chip processing of photonic quantum information [48-53] to high fidelities [54, 55], full reprogrammability for linear optical circuitry [56], and the integration of photon generation $[57,58]$ and detection $[59,60]$. Solid state single photon sources [61] and high efficiency detectors [62] have recently been demonstrated as a solution to achieving high numbers of photons. Ultimately, basic methods to correct for photon loss are likely to be required before photonic quantum simulations outperform classical algorithms [63], but the demands on error correction for specialised quantum simulators could be much lower than those for universal digital quantum simulators [64].

Here, our focus is on establishing programmable linear optical circuitry as a core capability for simulating the vibrational dynamics of the atoms within molecules. Our first set of demonstrations, in the harmonic approximation, simulates the evolution of single and multiple excitations for a range of four-atom molecules, and goes on to simulate coherent and incoherent energy transfer in $\mathrm{N}$-methylacetamide (NMA). Using $\mathrm{H}_{2} \mathrm{O}$ as an example, we show how to simulate thermal relaxation using ancillary optical modes, and how to include anharmonicities in the simulation model through measurement-induced nonlinearities. Finally, we show how our methods can be used as a testbed for control theory in molecules by placing our simulator in a closed loop adaptive feedback protocol, to find initial states which result in a particular dissociation pathway of $\mathrm{NH}_{3}$. Overall, we demonstrate a versatile simulator that can be rapidly reprogrammed to compare behaviour between different molecules, for a given physical model.

\section{Simulation procedure}

The procedure for simulating the vibrational quantum dynamics of a molecular system in the harmonic approximation begins with a calculation of the Hessian matrix, which is the second derivative of the electronic energy with respect to the nuclear coordinates at equilibrium geometry. (Note that this can be calculated practically for molecules with hundreds of vibrational modes, using standard techniques from quantum chemistry [65].) Diagonalising the Hessian in mass-weighted coordinates, and neglecting the eigenmodes related to molecular translation and rotation, provides the vibrational spectrum (eigenvalues) and normal modes (eigenvectors) of the system, which define a Hamiltonian of independent quantum harmonic oscillators $\hat{H}=\sum_{i} \hbar \omega_{i} a_{i}^{\dagger} a_{i}$ (omitting zero point energy).

The spatial localisation of vibrational energy is important for understanding many molecular phenomena such as energy transport and dissociation. We therefore consider a basis transformation, $a_{i}^{\dagger} \rightarrow \sum_{k} U_{k i}^{L} a_{k}^{\dagger}$, from the collective normal modes to a set of modes which are localised around a single atomic site or chemical bond. These localised modes are found using a Pipek-Mezey type method [66] by numerically maximising the sum of the squares of kinetic energy contributions from each nucleus. Dynamics in the localised basis can then be simulated via the model Hamiltonian $\hat{H}_{L}=\sum_{k, j} H_{k j}^{L} a_{k}^{\dagger} a_{j}$ where $H_{k j}^{L}=\sum_{i} \hbar \omega_{i} U_{k i}^{L} \overline{U_{j i}^{L}}$.

This general model can be directly simulated for $m$ vibrational modes of any given molecule with a linear optical chip that can be programmed to implement any unitary operation over $m$ optical modes [56, 67, 68]. Reconfiguring such a device to implement the transfer matrix $U\left(t_{i}\right)=\exp \left(-i H^{L} t_{i} / \hbar\right)$ for a series of timesteps $\left\{t_{i}\right\}$ allows the efficient simulation of the Hamiltonian $\hat{H}_{L}$ on any initial multimode vibrational state via its mapping to a multi-mode optical input state. Here we use a silica-on-silicon integrated photonic chip that is fully programmable over 6 waveguides via 30 thermo-optic phase shifters [56], to perform molecular simulations of up to six-mode vibrational systems. We simulate initial states of up to four vibrational quanta, with states of up to four single photons, produced from spontaneous parametric downconversion (SPDC) sources. Photons are detected with single photon counting modules. The number and pattern of photons collected at the output of the optical modes for each circuit configuration are governed by the probabilities for the molecule to be found in the corresponding vibrational states, at the corresponding timestep.

\section{Vibrational dynamics of four-atom molecules}

As a first example, we consider Thioformaldehyde $\left(\mathrm{H}_{2} \mathrm{CS}\right)$, a key molecule for spectroscopic experiments [69], which is shown in Fig. 1(a) with its normal mode spectrum. The 6 localised vibrational modes of $\mathrm{H}_{2} \mathrm{CS}$ comprise two $\mathrm{CH}$ stretch modes, two $\mathrm{CH}$ bend modes, a CS stretch mode, and a wagging mode, which are mapped to our photonic chip from the normal mode basis, as described above and conceptually depicted in Fig. 1(b). We initialised the simulation for the state $|\mu\rangle \sim \mu\left|1_{\mathrm{CHs} 1}, 1_{\mathrm{CHs} 2}\right\rangle+\mu^{2} / 2\left|2_{\mathrm{CHs} 1}, 2_{\mathrm{CHs} 2}\right\rangle$, of multiple excitations superposed over the two $\mathrm{CH}$ stretch modes, 
(a) $\mathrm{H}_{2} \mathrm{CS}$
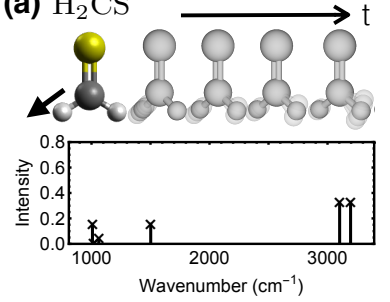

二 (b)

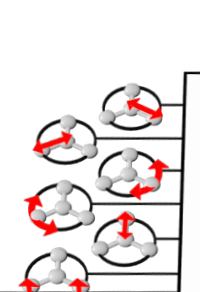

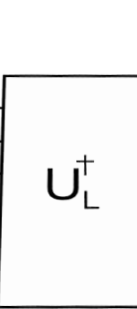
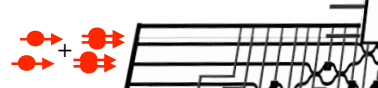

exis

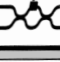

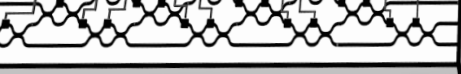
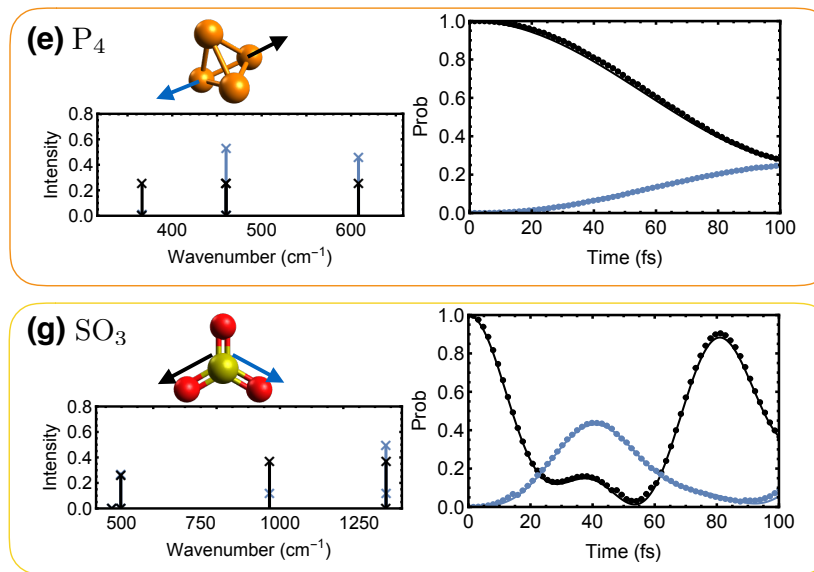
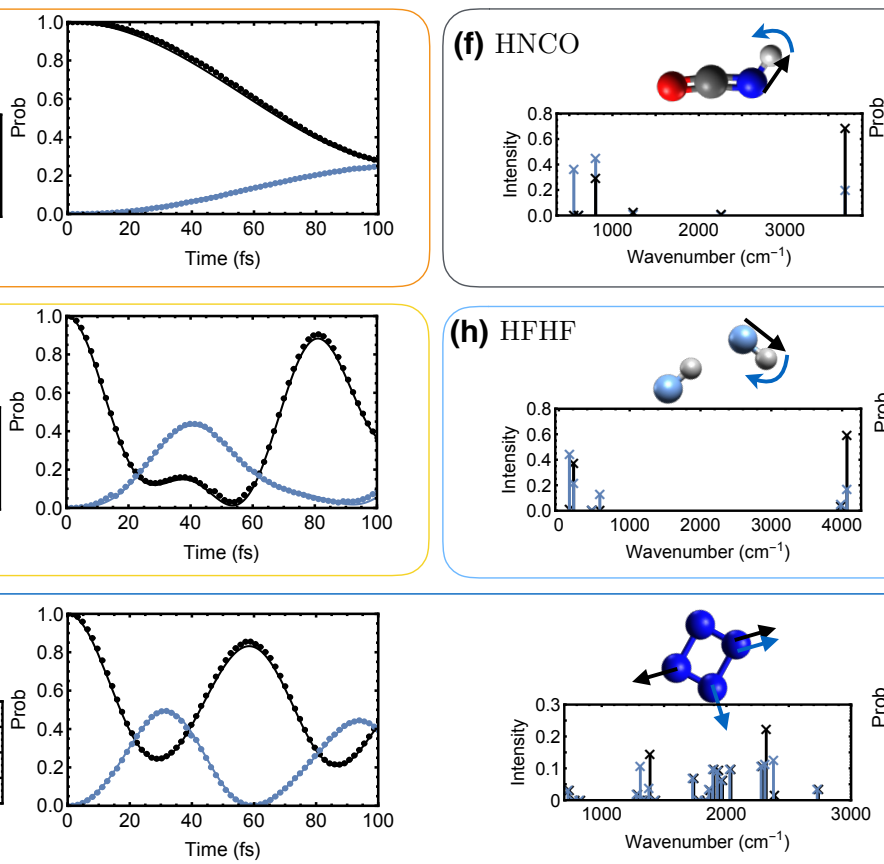

(c)

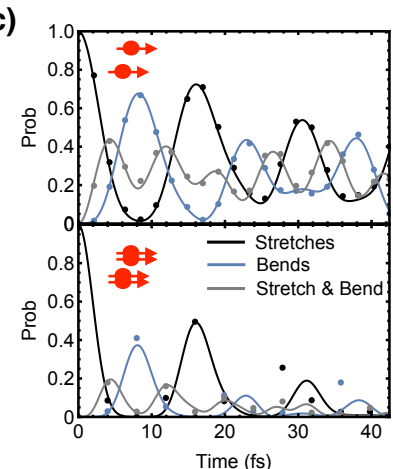

(d)

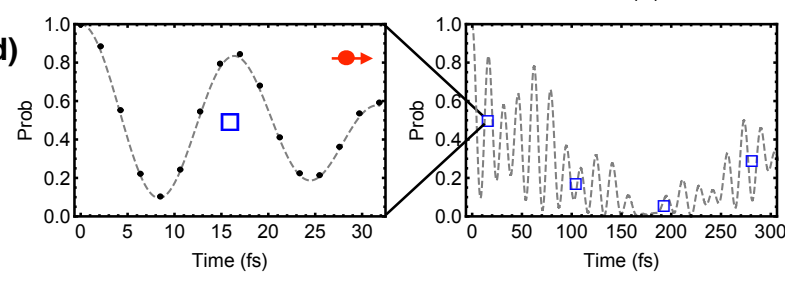

(h) HFHF
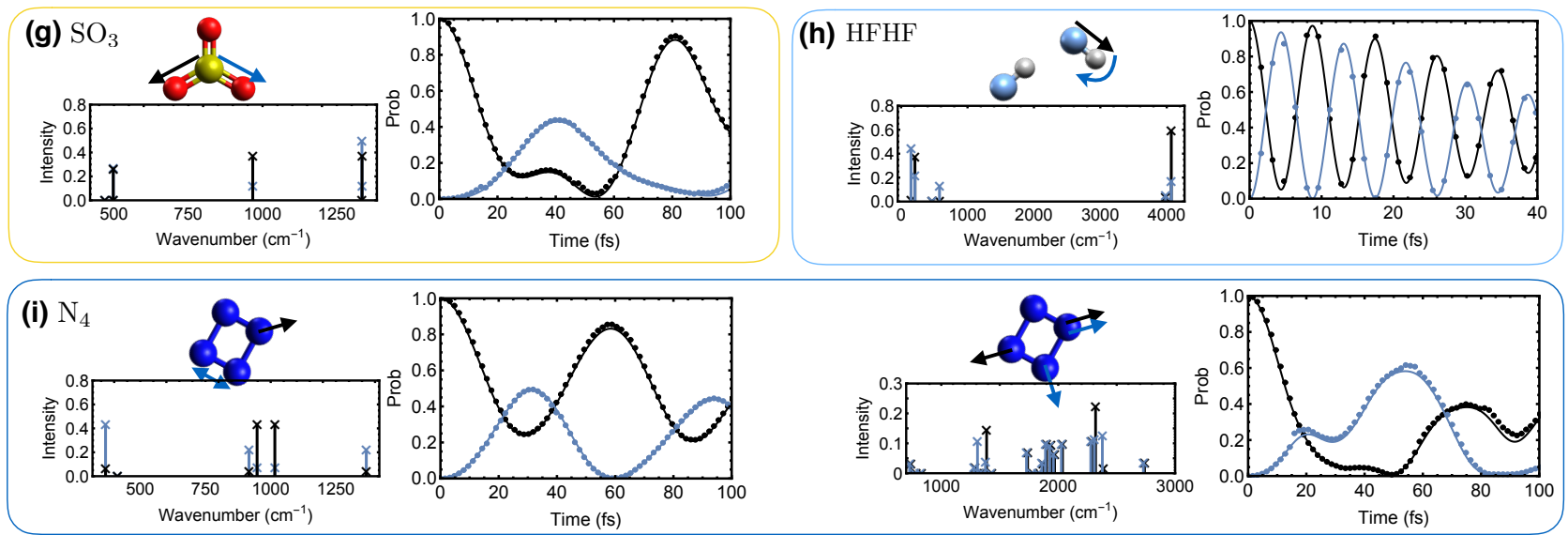

FIG. 1. Simulating the vibrational dynamics of four atom molecules in the harmonic approximation. (a) The eigenspectrum for $\mathrm{H}_{2} \mathrm{CS}$ is used to construct a molecular model of normal modes. (b) These are mapped to a Hamiltonian of local vibrational modes and a unitary operator that is implemented with our photonic chip for a sequence of time-steps. (c) An initial superposition of 2 and 4 excitations evolving in the localised stretch modes is simulated by injecting a two-mode squeezed vacuum state into the corresponding optical modes and collecting photon statistics for the sequence of simulated timesteps. Top and bottom panels show results for the two and four excitation subspaces respectively. (d) Simulations on two timescales for the evolving probability of a single excitation to remain in a $\mathrm{CH}$ stretch mode. Blue squares represent the mean probability over a $30 \mathrm{fs}$ window (as per left panel). (e-i) The simulated evolution of a single excitation in $\mathrm{P}_{4}, \mathrm{SO}_{3}, \mathrm{HNCO}$ and HFHF between a local stretch mode (black) and another coupled local mode (blue). The local modes are represented diagramatically alongside the spectral intensities of the normal modes involved. For $\mathrm{N}_{4}$, results are also shown for the evolution of two excitations. All data is plotted together with ideal theoretical curves; error bars displaying s.d. from Poissonian statistics are very small.

by injecting the two-mode squeezed vacuum state produced by an SPDC source, into the two waveguides corresponding to the $\mathrm{CH}$ stretch modes. Photons were collected over a series of circuit configurations which cor- respond to timesteps of the $\mathrm{H}_{2} \mathrm{CS}$ local-basis Hamiltonian. Fig. 1(c) displays the experimentally simulated evolution of the probabilities for excitations to be found in the $\mathrm{CH}$ stretch modes only, in the $\mathrm{CH}$ bend modes 


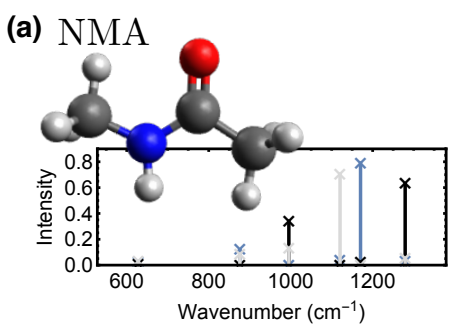

(c)

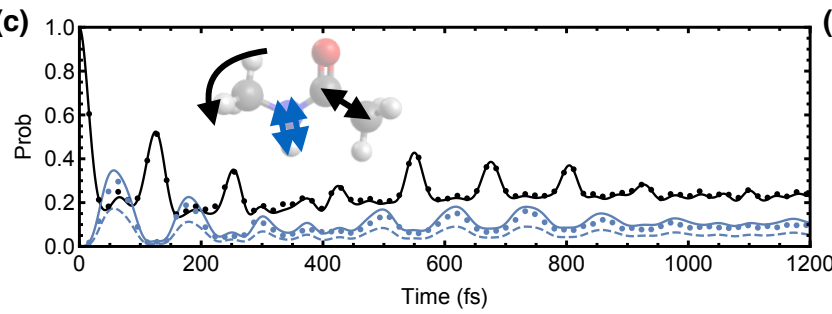

(b)

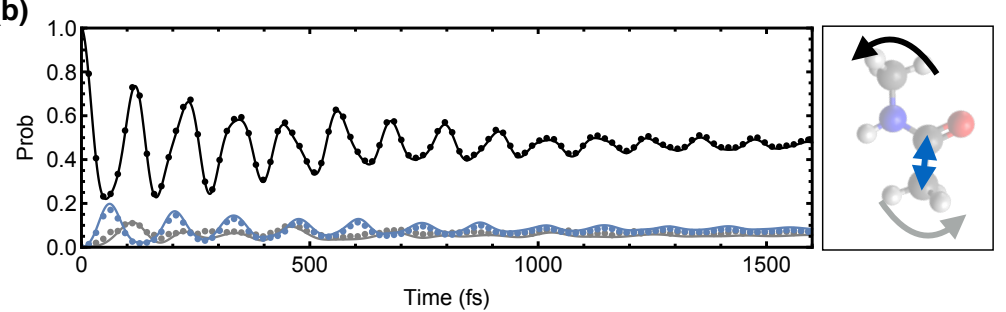

(d)

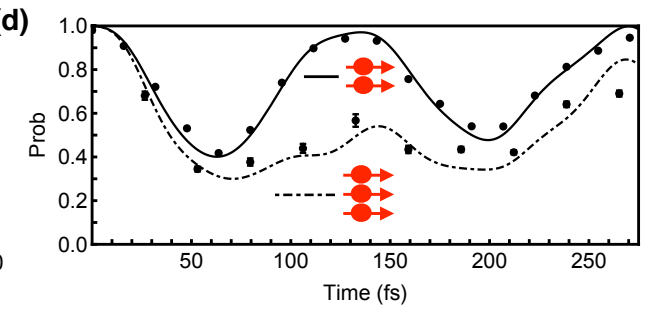

FIG. 2. Quantum energy transfer and dephasing in NMA. (a) A six-mode vibrational subspace of the NMA molecule is considered with the spectral components of three localised modes colour coded as per the arrows in (b). (b) Experimental simulation results for the probability of a single excitation (black points) initially in a local rocking mode (black arrow) at one end of the molecule and its transfer (blue and grey points) to local modes at the opposite end (blue and grey arrows) when subject to a dephasing channel with $T_{2}=0.53 p s$. (c) Experimental simulation results for the evolution of a two-excitation state (black points) that is initially in separate local modes (black arrows), and its probability (blue points) to be found bunched in the NH stretch mode (blue arrows) under the same dephasing channel. Solid lines represent theory. The dashed blue line plots a theoretical curve for distinguishable (or classical) excitations to be found bunched in the NH stretch mode. (d) Experimental simulation results for the total probability to measure a fully anti-bunched state of two excitations with the same intial state as (c) (black points with solid black theory curve) and to measure a fully anti-bunched state of three excitations initialised in the modes shown in (b) (black points with dashed theory curve). All error bars represent s.d. estimates from Poissonian statistics.

only, and shared between these stretches and bends for the two-excitation (upper panel) and four-excitation subspace (lower panel).

Dynamics in the two-excitation subspace involve both excitations oscillating between stretch and bend modes via the intermediate state where one excitation is in each of the subspaces. The $L^{1}$ distance $\mathcal{D}(\mathbf{p}, \mathbf{q})=$ $\frac{1}{2} \sum_{i}\left|p_{i}-q_{i}\right|$ between the results for an experimentally simulated timestep and the ideal distribution, is averaged over all timesteps to give $\overline{\mathcal{D}}=0.06 \pm 0.03$. In the fourexcitation subspace, where both of the stretch modes are initially doubly occupied, the experimentally simulated evolution of probabilities are shown for both stretch modes to remain doubly occupied, both bend modes to become doubly occupied, and for combinations of one doubly occupied stretch mode and one doubly occupied bend mode. The apparent damping of the oscillatory behaviour between these probabilities is attributable to the combinatorially growing space of multiple excitations available to the evolving state. The distance between the experimentally simulated and ideal evolutions for the full four photon distributions, averaged over all timesteps, is $\overline{\mathcal{D}}=0.16 \pm 0.07$. The full distributions for these and all succeeding experiments can be found in the appendix.

Because time is a programmable parameter in our simulator, we are able to probe dynamics at arbitrary evolution times. This can be exploited to study molecular vibrations whose evolution involves different timescales, such as the local $\mathrm{CH}$ stretch mode in $\mathrm{H}_{2} \mathrm{CS}$, which is a superposition of normal modes with lower and higher frequencies. The probability for a single excitation to remain localised in a $\mathrm{CH}$ stretch mode was simulated on two timescales that differ by an order of magnitude. Heralded single photons were injected into the mode corresponding to a local $\mathrm{CH}$ stretch, and the circuit was programmed to implement sets of unitary transformations that correspond to a short (30 fs) high resolution window, and a longer (300 fs) low resolution window whose behaviour can be observed by averaging over the high resolution windows. Fig. 1(d) displays the experimentally collected data for these simulations, which exhibit both high and lower frequency oscillations. Averaging over both evolutions gives a mean distance of $\overline{\mathcal{D}}=0.014 \pm 0.006$.

In general, molecules of $n$ atoms support $3 n-6$ vibrational modes, so a fully reprogrammable photonic chip of sufficient size can simulate the vibrational dynamics of any molecule in the harmonic approximation, using the techniques described here. Our six mode simulator can therefore explore the full space of vibrational dynamics for a general molecule of up to four atoms, as we demonstrate for $\mathrm{P}_{4}, \mathrm{SO}_{3}, \mathrm{HNCO}, \mathrm{HFHF}$ and $\mathrm{N}_{4}$, which comprise a wide range of molecular structures and energy scales.

Figures 1(e-i) show the time evolution of a single excitation initially prepared in a local stretch mode. The change in the occupation probability to a second, 
spectrally overlapped (coupled) local mode is plotted. We observe dynamics with varying characteristic times governed by the vibrational spectra of the molecules. Due to its geometry and bonding structure, $P_{4}$ has the longest period oscillations between opposing stretches with $\mathrm{SO}_{3}$ showing similar stretch mode coupling behaviour on shorter timescales. HNCO and HFHF in contrast display faster dynamics with increased mode coupling between hydrogen bond stretches and bends. Fig. 1(i) shows the dynamics of both a single excitation and two excitations initially prepared in stretch modes of $N_{4}$. The additional structure in the vibrational spectrum and the introduction of multi-photon quantum interference results in a more complex time dependence of the detection probabilities. The average $L^{1}$ distance over all these experiments is $\overline{\mathcal{D}}=0.022 \pm 0.007$, demonstrating accurate simulation over a wide range of molecules.

\section{Decoherence and energy transfer in NMA}

The flow of vibrational energy in molecules is a fundamental process for chemical reaction rates [70] and functionality in biomolecules [71], while control of intramolecular energy transfer could lead to new nanotechnologies such as molecular electronics [72]. The vibrational quantum dynamics of a molecule within an environment can be described by the interplay of coherent unitary evolution and incoherent dephasing resulting from random fluctuations of the vibrational frequencies - a process referred to as spectral diffusion. N-methylacetamide (NMA) is the simplest molecular model (Fig. 2(a)) of the peptide bond in proteins, where quantum coherence may play a role in energy transfer [73]. In this section, we simulate a model for intramolecular energy transport in NMA in the presence of dephasing.

We consider a subspace spanning 6 backbone vibrational modes, which support a basis of approximately localised vibrational modes, including two rocking modes (curved arrows in Fig. 2) and two stretch modes (straight arrows in Fig. 2). These six local modes are mapped to our photonic chip, such that the coherent time evolution of an excitation can be tracked, following the same procedure as described in the previous section. Uniform dephasing between all modes is achieved by a time-dependent statistical averaging over the set of experiments with transfer matrices $U\left(t_{i}, k\right)=$ $U_{L} Z(k) U_{L}^{\dagger} e^{-i H^{L} t_{i} / \hbar}$ where $Z(k)$ are Heisenberg-Weyl matrices and the average is taken over $k$ at each timestep. (See appendix for details.)

Using a single photon, we simulated the probability for a single excitation initialised in a local rocking mode at one end of the molecule to be transferred to two localised modes (a rocking mode and a $\mathrm{C}-\mathrm{C}$ stretch mode) at the opposite end of the molecule. The experimental results shown in Fig. 2(b) show dynamics that are initially oscillatory, with vibrational energy transfer between the rocking modes at either end of the molecule via an intermediate $\mathrm{C}-\mathrm{C}$ stretch. The increasing effect of the suppression of coherence from dephasing results in evolution towards a steady state. The peak probabilities for energy to be localised at either end of the molecule are higher under quantum coherent dynamics than under purely ballistic classical dynamics. The $T_{2}$ time constant of coherence decay used is $0.53 \mathrm{ps}$, though any time constant can be simulated by changing only the post-processing of data.

Generalising the simulation for initial states of multiple excitations allows us to investigate the interplay of dephasing and quantum interference for multi-excitation energy transport. Injecting one photon into the waveguide corresponding to the rocking mode and another photon into the waveguide corresponding to the $\mathrm{C}-\mathrm{C}$ stretch mode, which are each localised at opposite ends of the NMA molecule (black arrows in Fig. 2(c)), we simulated the change in the probability for this state, and the state in which both excitations 'bunch' in an $\mathrm{NH}$ stretch mode (double blue arrows in Fig. 2(c)). The results in Fig. 2(c) show more complex oscillatory transfer between these bunched and anti-bunched states, that again tends toward a steady state. However, after full dephasing has occurred, the probability for two excitations to be bunched in the NH stretch mode is twice as high for excitations that behave as indistinguishable bosonic particles than for excitations that behave as distinguishable or classical particles (e.g. two excitations that pass through the molecule at different times) whose theoretical probability is also plotted.

For a given molecule, the probability that no bunching occurs (multiple excitations are not localised around the same bond) generally decreases as the number of excitations increases [74]. In Fig. 2(d) the probability for the subspace of no-bunching events is simulated for two and three excitations under fully coherent dynamics. The initial state for the two-excitation evolution is the same as in the previous example; the initial state for the three-excitation evolution comprises an excitation in each of the local modes shown in Fig. 2(b). The average distances across all single, two and three excitation distributions in these examples are given by $0.017 \pm 0.005,0.05 \pm 0.01$ and $0.14 \pm 0.07$ respectively.

\section{Vibrational relaxation in liquid water}

We now consider extensions to the idealised model of uncoupled harmonic oscillators, to account for more realistic situations including energy dissipation and anharmonic potentials. We choose models for $\mathrm{H}_{2} \mathrm{O}$ to demonstrate our techniques for simulating these effects.

For a molecule that interacts with its environment, vibrational energy is exchanged via intra- and intermolecular coupling to other degrees of freedom, eventually leading to thermalisation. This process is known as vibrational relaxation, and its pathways for $\mathrm{H}_{2} \mathrm{O}$ remain an area of current investigation [75-77]. Here we simulate the relaxation of $\mathrm{H}_{2} \mathrm{O}$ via an amplitude damping model, depicted in Fig. 3(a).

In describing these open system dynamics, we consider a Lindblad master equation, resulting in a set of timedependent Kraus operators which can be simulated via 

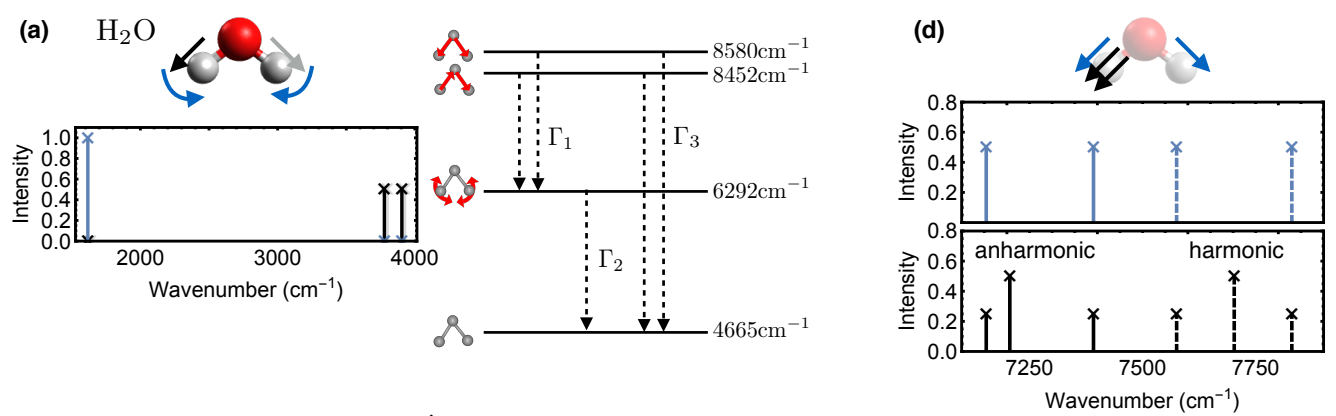

(b)
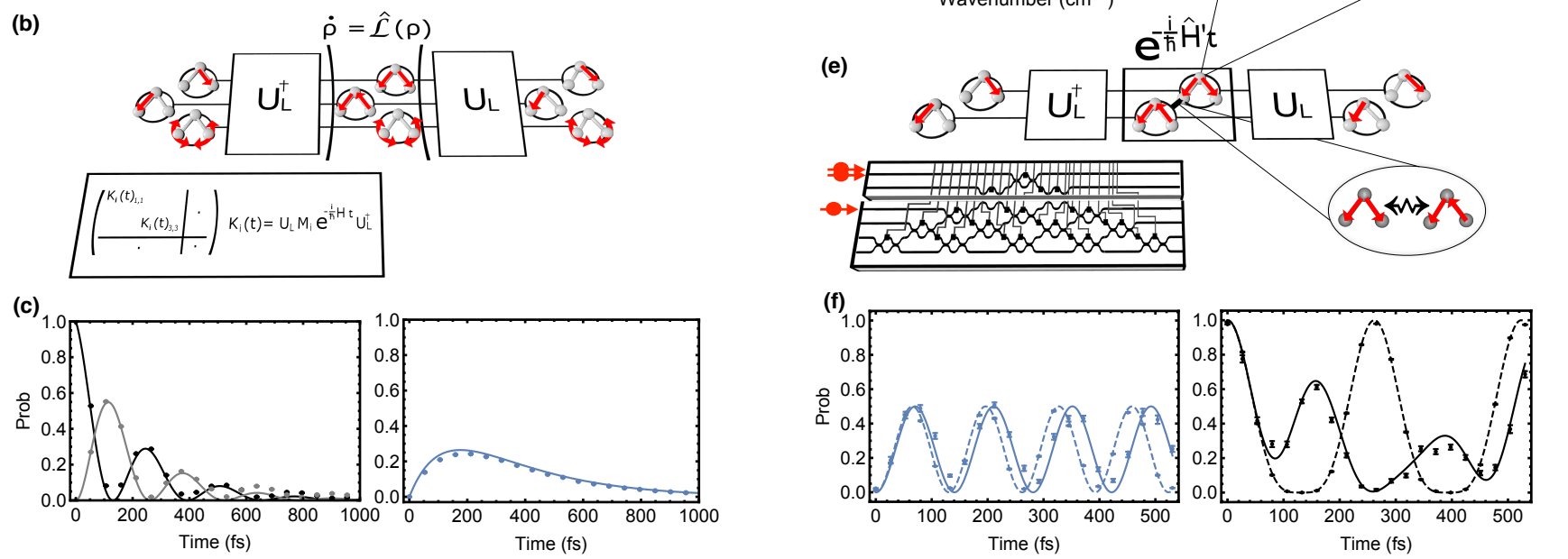

FIG. 3. Vibrational relaxation and anharmonic evolution for $\mathrm{H}_{2} \mathrm{O}$. (a) Energy level diagram for single excitation harmonic levels and ground state of $\mathrm{H}_{2} \mathrm{O}$ along with the spectral components of the two local $\mathrm{OH}$ stretch modes (black and grey) and the symmetric bend normal mode (blue). (b) The open system dynamics of vibrational relaxation can be simulated by statistical averaging of the evolution under a set of linear operators implemented via unitary dilation. (c) Experimental results for the simulated evolution of the probability for a single excitation, initially in one $\mathrm{OH}$ stretch mode (black points), to be found in the other stretch mode (grey points) and the symmetric bend (blue points) under relaxation dynamics. Solid lines are theoretical curves. (d) Spectrum of two excitations in bunched (black) and anti-bunched (blue) local stretch modes for a harmonic (dashed) and anharmonic model (solid). (e) The anharmonic evolution is implemented via a measurement-induced nonlinearity using an ancillary photon and modes. (f) Experimental results for the simulated evolution of two excitations initially bunched in local stretch modes to be found in the anti-bunched state (left panel) and the bunched state (right panel) under both models (dashed harmonic, solid anharmonic). All error bars represent s.d. estimates from Poissonian statistics.

an ensemble of transfer matrices. This evolution cannot be described as a convex sum of unitary evolutions as in the previous section, however the transfer matrices can be realised within a unitary matrix of twice the size, via unitary dilation [78]. Because $\mathrm{H}_{2} \mathrm{O}$ has three vibrational modes, its three dimensional (non-unitary) transfer matrices can be realised within a six dimensional unitary matrix, and can be implemented on our six mode chip, as shown in Fig. 3(b). Experimentally measured relaxation times $\left\{\Gamma_{i}\right\}$ for liquid water at room temperature in Ref. [76] were used in the model. (See appendix for details.)

We simulated the thermalisation of an excitation in a local $\mathrm{OH}$ stretch mode, via the symmetric bend normal mode, to its ground state of no excitations. Fig. 3(c) shows the probability of measuring the excitation in the two local stretch modes (left panel) and the symmetric bend (right panel). Oscillations between the two high energy stretch modes decay, as the population is transferred via the lower energy bend mode to the ground state. The average $L^{1}$ distance in these experiments was $\overline{\mathcal{D}}=0.024 \pm 0.007$.

\section{Anharmonic potentials in $\mathrm{H}_{2} \mathrm{O}$}

While the utility of the harmonic approximation makes it a mainstay model, the potential energy surfaces of real molecules are anharmonic, and we now consider simulations in this regime, depicted in Fig. 3(d). In addition to the second derivative in the Taylor expansion of the potential energy surface, as per the harmonic approximation, we now include all third derivatives and the semidiagonal quartic derivatives. Applying vibrational perturbation theory $[79,80]$ yields a new Hamiltonian

$$
\hat{H}_{a}=\hat{H}+\hbar \sum_{i \leq j} \frac{x_{i j}}{2} \sqrt{\omega_{i} \omega_{j}}\left(a_{i}^{\dagger} a_{i}+a_{j}^{\dagger} a_{j}+2 a_{i}^{\dagger} a_{j}^{\dagger} a_{i} a_{j}\right)
$$


(a)

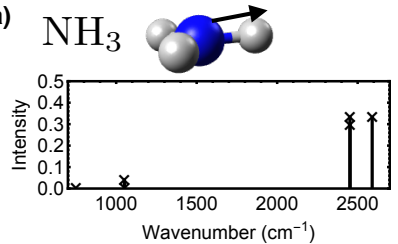

(b)

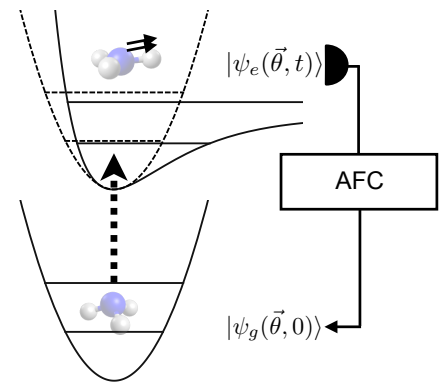

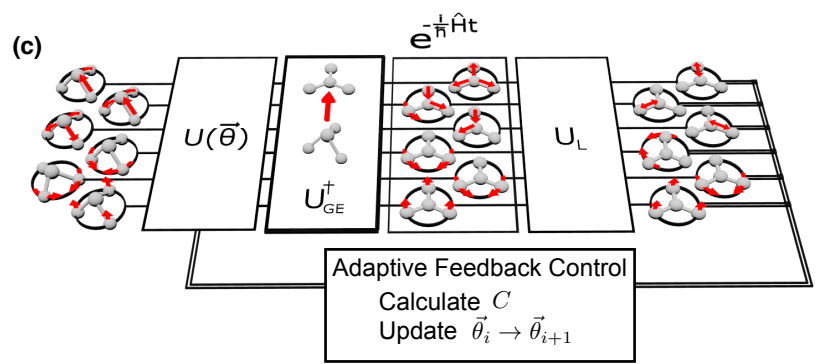

(d)

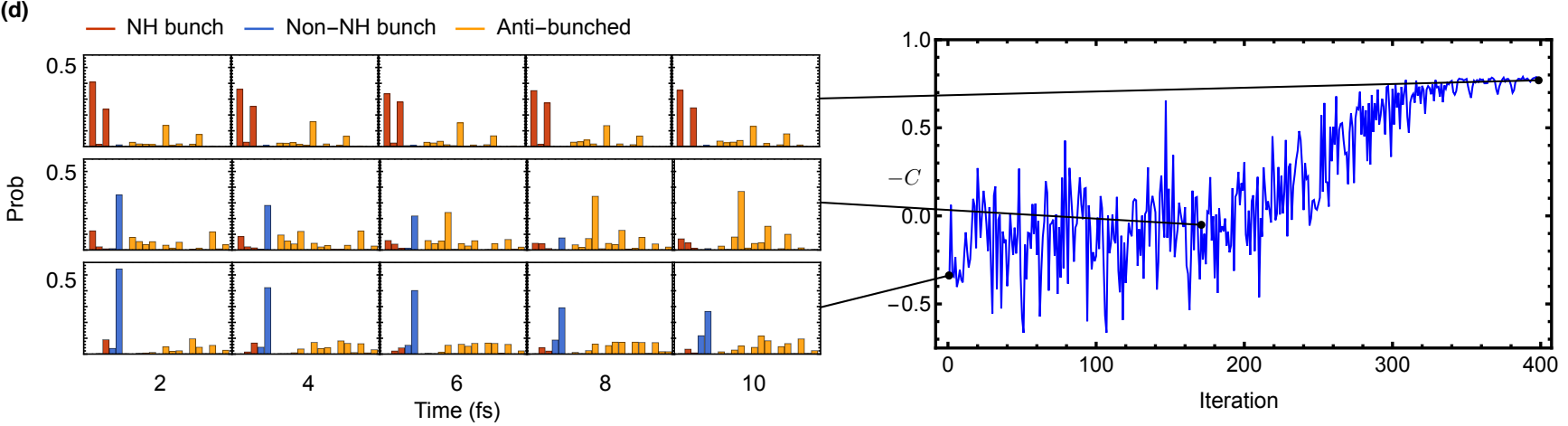

FIG. 4. Adaptive feedback control algorithm for a dissociation pathway in $\mathrm{NH}_{3}$. (a) The spectral decomposition of an $\mathrm{NH}$ stretch mode in the electronic excited state of $\mathrm{NH}_{3}$. (b) A two-excitation vibrational state, parameterised by $\vec{\theta}$, is initialised in the ground state vibrational modes of $\mathrm{NH}_{3}$. The electronic state is excited and the localisation of vibrational energy is measured over time. These measurements are used to feedback to the state preparation in order to increase energy localisation in NH stretch modes, promoting a particular disassociation pathway for this molecule. (c) This scenario is simulated via a parameterised unitary for state preparation $U(\vec{\theta})$, a transformation between the ground state and excited state modes $U_{\mathrm{GE}}^{\dagger}$, evolution under the excited state modes and measurement in a localised basis via $U_{L}$. The resulting photon statistics are fed back through an AFC algorithm to set $\vec{\theta}$ for the next iteration. (d) An example set of experimental results showing (left panel) the full distributions for bunching in the $\mathrm{NH}$ stretch modes (red), bunching in the remaining three localised modes (blue) and detection in anti-bunched patterns (yellow) for five timesteps at iteration numbers 1 (bottom), 175 (middle) and 399 (top). The right panel shows the measured cost function $-C$ at every iteration.

where $\hat{H}$ is the harmonic Hamiltonian and $x_{i j}$ are the perturbation theory coefficients.

Implementing this Hamiltonian requires interactions between photons - a key challenge in quantum information processing. Demonstrations of enhanced single photon interactions have required, for example, an artificial Kerr medium using superconducting circuits [81], fibre coupling a single atom and micro-resonantor [82], or coupling to a single quantum dot in a micropillar cavity[83]. Importantly, the interactions required to implement perturbative models like $\hat{H}_{a}$ can be weaker than the fully entangling operations and controlled $\pi$-phase gates used for universal quantum computing [84], with a potentially lower demand for reprogrammable nonlinear optics. Nonlinear operations in silicon based materials, such as integrated nonlinear Mach Zehnder interferometers in silicon [85] and large phase shifts in silicon nitride [86] have involved strong laser light, while only very small Kerr nonlinearities have been observed at the single or few-photon level using silica photonic crystal fibres [87], atoms in hollow fibres [88] and laser-cooled atoms [89]. However, novel microcavity designs to highly concentrate light present new possibilities for strong single-photon Kerr nonlinearities [90].

Here, instead, we demonstrate an approach based on measurement-induced nonlinearities, which are in principle scalable for all-linear-optical quantum computing, though involve a large overhead. It is possible to implement a conditional $\pi$-phase shift on a two-photon Fock state using an ancillary photon, and additional optical modes [91]. Using newly derived nonlinear phase-shift (NPS) gates, we are able to implement arbitrary phase shifts between the zero, one and two Fock states of an optical mode. (See appendix for details.)

We simulate and compare harmonic and anharmonic models of vibration for $\mathrm{H}_{2} \mathrm{O}$, restricting to the subspace of stretch modes. Two photons injected together into the top mode of the chip serves to simulate two excitations initialised in a superposition of the harmonic model eigenstates corresponding to a local $\mathrm{OH}$ stretch. As shown in Fig. 3(e), when simulating the anharmonic model, this input state can now be understood as the same superposition of the new energy eigenstates of $\hat{H}_{a}$, while a third photon injected into the third optical mode serves as the ancillary system. 
Fig. 3(f) shows the results for simulating the probabilties for these two vibrational excitations to remain bunched or anti-bunch, under both the harmonic model, as before, and the anharmonic model using the NPS gate. The difference in the detection patterns between the two models, $\hat{H}$ and $\hat{H}_{a}$, is a function of their different spectra, shown in Fig. 3(d). The probability to detect a single excitation in each of the modes (i.e. anti-bunched), shown in the left panel of Fig. 3(f), acquires a simple frequency shift for the anharmonic evolution corresponding to the adjusted energy levels, as seen in the top panel of Fig. 3(d). In contrast, the probabilities for the state to remain doubly occupied display markedly different dynamics between the harmonic and anharmonic cases, as shown in the right panel of Fig. 3(f). This is a result of the three vibrational eigenstates no longer being equally spaced in energy, as seen in the bottom panel of Fig. 3(d), introducing new frequencies in the evolution. For this set of experiments, the average distances between the ideal and experimental distributions for the harmonic and anharmonic cases are $0.02 \pm 0.01$ and $0.06 \pm 0.02$ respectively.

\section{Quantum simulation with adaptive feedback}

Adaptive feedback control (AFC) is a practical laboratory technique for finding optimal control fields for molecules [13-16]. Starting with a random selection of pulse shapes, the measured outcome from each control setting is evaluated with respect to a predefined goal by an algorithm that searches the available space of control parameters and iterates towards to an optimal solution. AFC naturally incorporates laboratory constraints to design control fields that would not be found either analytically or through numerical simulation. Nevertheless, models idealised for quantum simulation could help to identify new possibilities for molecular control, allow their comparison over a large number of molecules, and include quantum control fields.

Our goal is to use our simulator with an adaptive feedback loop from its quantum measurement statistics to design initial quantum states for a molecule that maximally achieve a particular task over a period of evolution. Our example molecule is ammonia, $\mathrm{NH}_{3}$, a prototype for studying dissociation, including vibrationally mediated pathways, in which the states of its products, $\mathrm{NH}_{2}+\mathrm{H}$ depend on the prior vibrational state in the ground electronic state [17].

Our model, shown in Fig. 4(b), simulates the preparation of a vibrational state in the electronic ground state of the molecule; we then obtain the vibrational state that results from an electronic excitation by projecting the vibrational modes of the ground state onto the vibrational modes of the excited state. We approximate this projection by a unitary transformation between the modes $U_{\mathrm{GE}}$, though note that this transformation can in general be achieved via single mode squeezing, displacement, and linear optical transformations [92]. The evolution of the vibrational state of the electronically excited molecule is simulated under the harmonic approximation Hamiltonian for the normal modes. Measuring the evolved state in a localised basis, as before, identifies three local $\mathrm{NH}$ stretch modes.

The specific aim of this simulation, schematically depicted in Fig. 4(c), is to let an AFC algorithm find initial states of two vibrational excitations (in the electronic ground state molecule) which result in a maximal total probability to bunch in any of the three $\mathrm{NH}$ stretch modes (of the electronically excited molecule) over the first 10fs of evolution, which we associate with a preferred dissociation pathway, whilst suppressing other bunched events which we associate with other pathways. The algorithm begins with a trial state of two photons that simulates two excitations superposed randomly over 5 of the normal vibrational modes (the 6th mode is essentially uncoupled from the rest). State preparation, parameterised by $U(\vec{\theta})$, is iteratively optimised by programming the simulator to implement the transfer matrix, $U\left(\vec{\theta}, t_{i}\right)=U_{L} e^{-i H t_{i} / \hbar} U_{\mathrm{GE}}^{\dagger} U(\vec{\theta})$, where $U_{\mathrm{GE}}$ relates to the transformation between the ground and excited state normal modes and $U_{L}$ relates to the transformation between the excited state normal and local modes.

An example experimental trial is shown in Fig. 4(d). A Nelder-Mead simplex method is used to minimise the cost function $C=-\alpha \sum_{i} w_{i} \Delta p_{i} \in[1,-1]$, where $\Delta p_{i}$ is the difference between the probability of bunching in the $\mathrm{NH}$ stretch modes and the remaining modes at timestep $i, w_{i}$ are weighting factors, and $\alpha$ is a normalisation factor (see appendix for details). The final value in Fig. 4(d) is $C=-0.771$, starting from a random initial state with $C=+0.337$. In all, this procedure involved the collection of measurement statistics for almost 2000 unique settings of the photonic chip. This experiment was repeated with six random initial states, all achieving similar final values of the cost function with a mean $\bar{C}=-0.845$ (see appendix for details).

\section{Discussion}

We have introduced integrated photonics as a platform for simulating the vibrational quantum dynamics of molecules within the harmonic, perturbatively anharmonic and Linblad models, demonstrating a core mapping in which a photonic chip plays the role of a programmable molecule. Scaling up and extending these techniques to more involved Hamiltonians with highly anharmonic atomic potentials and electronic degrees of freedom, and to realise an advantage over classical simulation techniques [63], present important and interesting research directions.

Particularly relevant device errors that must be addressed are those of inevitable flaws in circuitry fabrication and operation, photon distinguishability, and photon loss. Although the precision required in the setting of any individual circuit parameter must necessarily increase with dimension [93, 94], linear optical elements with extinction ratios of over $60 \mathrm{~dB}$ have been demonstrated $[53,55]$. In addressing the task of reducing distinguishability between independent photons, visibilities of $95 \%$ have been reported for on-chip sources [95], and 
visibilities $>90 \%$ have been reported for photons from widely separated time bins of a solid state photon source [96]. While ultra low loss integrated circuitry has been demonstrated [97], photon loss is a fundamental error in photonics; yet basic methods that alleviate some of this error would provide significant improvements in rates for the class of experiments demonstrated here.

The development of programmable nonlinear optics at the quantum level is a key functionality for quantum technologies and remains a major challenge for the field, however, we have shown that weak nonlinearities provide a sufficient augmentation to simulate richer molecular models. With modest progress in these areas, our approach could yield an early class of practical quantum simulations that operate beyond current classical limits. It might then be possible to discover new possibilities for molecular control with quantum states of light, including new dissociation pathways and molecular conformations.
[1] B. Ulmann, Analog Computing (Oldenbourg Wissenschaftsverlag, 2013).

[2] C. Care, Technology for Modelling: Electrical Analogies, Engineering Practice, and the Development of Analogue Computing (Springer-Verlag London, 2010).

[3] F. Gatti, Molecular Quantum Dynamics (SpringerVerlag Berlin Heidelberg, 2014).

[4] C. Brif, R. Chakrabarti, and H. Rabitz, New Journal of Physics 12, 075008 (2010).

[5] R. P. Feynman, Int. J. Theor. Phy. 21, 467 (1982).

[6] S. Lloyd, Science 273 (1996).

[7] I. Buluta and F. Nori, Science 326, 108 (2009).

[8] T. D. Ladd, F. Jelezko, R. Laflamme, Y. Nakamura, C. Monroe, and J. L. O'Brien, Nature 464, 45 (2010).

[9] A. Aspuru-Guzik and P. Walther, Nat. Phys. 8, 285 (2012).

[10] I. M. Georgescu, S. Ashhab, and F. Nori, Rev. Mod. Phys. 86, 153 (2014).

[11] S. Shi, A. Woody, and H. Rabitz, The Journal of Chemical Physics 88, 6870 (1988).

[12] M. Shapiro and P. Brumer, Reports on Progress in Physics 66, 859 (2003).

[13] H. Rabitz, R. de Vivie-Riedle, M. Motzkus, and K. Kompa, Science 288, 824 (2000).

[14] A. Assion, T. Baumert, M. Bergt, T. Brixner, B. Kiefer, V. Seyfried, M. Strehle, and G. Gerber, Science 282, 919 (1998).

[15] R. J. Levis, G. M. Menkir, and H. Rabitz, Science 292, 709 (2001).

[16] T. Brixner, N. H. Damrauer, P. Niklaus, and G. Gerber, Nature 414, 57 (2001).

[17] M. L. Hause, Y. H. Yoon, and F. F. Crim, The Journal of Chemical Physics 125 (2006).

[18] D. Brinks, F. D. Stefani, F. Kulzer, R. Hildner, T. H. Taminiau, Y. Avlasevich, K. Mullen, and N. F. van Hulst, Nature 465, 905 (2010).

[19] A. Alnaser, M. Kbel, R. Siemering, B. Bergues, N. G. Kling, K. Betsch, Y. Deng, J. Schmidt, Z. Alahmed, A. Azzeer, J. Ullrich, I. Ben-Itzhak, R. Moshammer, U. Kleineberg, F. Krausz, R. de Vivie-Riedle, and M. Kling, Nat Comms 5 (2014), 10.1038/ncomms4800.

[20] X. Tong, A. H. Winney, and S. Willitsch, Phys. Rev. Lett. 105, 143001 (2010).

[21] M. McDonald, B. H. McGuyer, F. Apfelbeck, C. H. Lee, I. Majewska, R. Moszynski, and T. Zelevinsky, Nature 534, 122 (2016).

[22] T. L. Cocker, D. Peller, P. Yu, J. Repp, and R. Huber, Nature 539, 263 (2016).

[23] B. Wolter, M. G. Pullen, A.-T. Le, M. Baudisch, K. Doblhoff-Dier, A. Senftleben, M. Hemmer,
C. D. Schröter, J. Ullrich, T. Pfeifer, R. Moshammer, S. Gräfe, O. Vendrell, C. D. Lin, and J. Biegert, Science 354, 308 (2016).

[24] E. S. Polzik, J. Carri, and H. J. Kimble, Phys. Rev. Lett. 68, 3020 (1992).

[25] M. A. Taylor, J. Janousek, V. Daria, J. Knittel, B. Hage, BachorHans-A., and W. P. Bowen, Nat Photon 7, 229 (2013).

[26] C. H. H. Schulte, J. Hansom, A. E. Jones, C. Matthiesen, C. Le Gall, and M. Atature, Nature 525, 222 (2015).

[27] J. B. Clark, F. Lecocq, R. W. Simmonds, J. Aumentado, and J. D. Teufel, Nature 541, 191 (2017).

[28] X.-L. Chu, S. Götzinger, and V. Sandoghdar, Nat Photon 11, 58 (2017).

[29] M. G. Raymer, A. H. Marcus, J. R. Widom, and D. L. P. Vitullo, The Journal of Physical Chemistry B 117, 15559 (2013).

[30] K. E. Dorfman, F. Schlawin, and S. Mukamel, Rev. Mod. Phys. 88, 045008 (2016).

[31] F. Schlawin and A. Buchleitner, New Journal of Physics 19, 013009 (2017).

[32] A. Pe'er, B. Dayan, A. A. Friesem, and Y. Silberberg, Phys. Rev. Lett. 94, 073601 (2005).

[33] C. Bernhard, B. Bessire, T. Feurer, and A. Stefanov, Phys. Rev. A 88, 032322 (2013).

[34] J. M. Lukens, A. Dezfooliyan, C. Langrock, M. M. Fejer, D. E. Leaird, and A. M. Weiner, Opt. Lett. 38, 4652 (2013).

[35] M. Karpiński, M. Jachura, L. J. Wright, and B. J. Smith, Nat Photon 11, 53 (2017).

[36] L. Valiant, Theoretical Computer Science 8, 189 (1979).

[37] S. Aaronson and A. Arkhipov, Theory of Computing 9, 143 (2013).

[38] D. W. Berry, A. M. Childs, R. Cleve, R. Kothari, and R. D. Somma, Phys. Rev. Lett. 114, 090502 (2015).

[39] B. P. Lanyon, C. Hempel, D. Nigg, M. Müller, R. Gerritsma, F. Zähringer, P. Schindler, J. T. Barreiro, M. Rambach, G. Kirchmair, M. Hennrich, P. Zoller, R. Blatt, and C. F. Roos, Science 334, 57 (2011), http://science.sciencemag.org/content/334/6052/57.full.pdf.

[40] I. Kassal, S. P. Jordan, P. J. Love, M. Mohseni, and A. Aspuru-Guzik, Proceedings of the National Academy of Sciences 105, 18681 (2008), http://www.pnas.org/content/105/48/18681.full.pdf.

[41] D. A. Lidar and H. Wang, Phys. Rev. E 59, 2429 (1999).

[42] E. T. Campbell, B. M. Terhal, and C. Vuillot, Nature 549, $172(2017)$.

[43] D. Wecker, B. Bauer, B. K. Clark, M. B. Hastings, and M. Troyer, Phys. Rev. A 90, 022305 (2014). 
[44] J. R. McClean, J. Romero, R. Babbush, and A. AspuruGuzik, New Journal of Physics 18, 023023 (2016).

[45] A. Kandala, A. Mezzacapo, K. Temme, M. Takita, M. Brink, J. M. Chow, and J. M. Gambetta, Nature 549, 242 (2017).

[46] A. Y. Smirnov, S. Savel'ev, L. G. Mourokh, and F. Nori, EPL (Europhysics Letters) 80, 67008 (2007).

[47] R. Babbush, P. J. Love, and A. Aspuru-Guzik, Scientific Reports 4, 6603 (2014).

[48] A. Politi, M. J. Cryan, J. G. Rarity, S. Yu, and J. L. O'Brien, Science 320, 646 (2008).

[49] J. L. O'Brien, A. Furusawa, and J. Vuckovic, Nat Photon 3, 687 (2009).

[50] D. Bonneau, J. W. Silverstone, and M. G. Thompson, "Silicon quantum photonics," in Silicon Photonics III: Systems and Applications (Springer Berlin Heidelberg, Berlin, Heidelberg, 2016) pp. 41-82.

[51] A. Crespi, R. Ramponi, R. Osellame, L. Sansoni, I. Bongioanni, F. Sciarrino, G. Vallone, and P. Mataloni, Nat. Comms. 2, 566 (2011).

[52] B. J. Metcalf, J. B. Spring, P. C. Humphreys, N. Thomas-Peter, M. Barbieri, W. S. Kolthammer, X.M. Jin, N. K. Langford, D. Kundys, J. C. Gates, B. J. Smith, P. G. R. Smith, and I. A. Walmsley, Nat Photon 8, 770 (2014).

[53] N. C. Harris, G. R. Steinbrecher, M. Prabhu, Y. Lahini, J. Mower, D. Bunandar, C. Chen, F. N. C. Wong, T. Baehr-Jones, M. Hochberg, S. Lloyd, and D. Englund, Nat Photon 11, 447 (2017).

[54] A. Laing, A. Peruzzo, A. Politi, M. R. Verde, M. Halder, T. C. Ralph, M. G. Thompson, and J. L. O'Brien, Appl. Phys. Lett. 97, 211109 (2010).

[55] C. M. Wilkes, X. Qiang, J. Wang, R. Santagati, S. Paesani, X. Zhou, D. A. B. Miller, G. D. Marshall, M. G. Thompson, and J. L. O'Brien, Opt. Lett. 41, 5318 (2016).

[56] J. Carolan, C. Harrold, C. Sparrow, E. Martín-López, N. J. Russell, J. W. Silverstone, P. J. Shadbolt, N. Matsuda, M. Oguma, M. Itoh, G. D. Marshall, M. G. Thompson, J. C. F. Matthews, T. Hashimoto, J. L. O'Brien, and A. Laing, Science 349, 711 (2015).

[57] J. W. Silverstone, D. Bonneau, K. Ohira, N. Suzuki, H. Yoshida, N. Iizuka, M. Ezaki, C. M. Natarajan, M. G. Tanner, R. H. Hadfield, V. Zwiller, G. D. Marshall, J. G. Rarity, J. L. O'Brien, and M. G. Thompson, Nat Photon 8, 104 (2014).

[58] M. Arcari, I. Söllner, A. Javadi, S. Lindskov Hansen, S. Mahmoodian, J. Liu, H. Thyrrestrup, E. H. Lee, J. D. Song, S. Stobbe, and P. Lodahl, Phys. Rev. Lett. 113, 093603 (2014).

[59] F. Najafi, J. Mower, N. C. Harris, F. Bellei, A. Dane, C. Lee, X. Hu, P. Kharel, F. Marsili, S. Assefa, K. K. Berggren, and D. Englund, Nat. Comms. 6, 5873 (2015).

[60] C. Schuck, X. Guo, L. Fan, X. Ma, M. Poot, and H. X. Tang, Nat. Comms. 7, 10352 (2016).

[61] H. Wang, Y. He, Y.-H. Li, Z.-E. Su, B. Li, H.-L. Huang, X. Ding, M.-C. Chen, C. Liu, J. Qin, J.-P. Li, Y.-M. He, C. Schneider, M. Kamp, C.-Z. Peng, S. Höfling, C.-Y. Lu, and J.-W. Pan, Nat Photon 11, 361 (2017).

[62] F. Marsili, V. B. Verma, J. A. Stern, S. Harrington, A. E. Lita, T. Gerrits, I. Vayshenker, B. Baek, M. D. Shaw, R. P. Mirin, and S. W. Nam, Nat Photon 7, 210 (2013).
[63] A. Neville, C. Sparrow, R. Clifford, E. Johnston, P. M. Birchall, A. Montanaro, and A. Laing, Nat. Phys. , (2017).

[64] T. Cubitt, A. Montanaro, and S. Piddock, "Universal quantum hamiltonians," (2017), arXiv:1701.05182.

[65] D. H. Friese, C. Httig, and J. Komann, Journal of Chemical Theory and Computation 9, 1469 (2013).

[66] C. R. Jacob and M. Reiher, The Journal of Chemical Physics 130, 084106 (2009).

[67] M. Reck, A. Zeilinger, H. J. Bernstein, and P. Bertani, Phys. Rev. Lett. 73, 58 (1994).

[68] W. R. Clements, P. C. Humphreys, B. J. Metcalf, W. S. Kolthammer, and I. A. Walmsley, Optica 3, 1460 (2016).

[69] D. J. Clouthier and D. A. Ramsay, Annual Review of Physical Chemistry 34, 31 (1983).

[70] M. Gruebele and P. G. Wolynes, Accounts of Chemical Research 37, 261 (2004).

[71] D. M. Leitner, Annual Review of Physical Chemistry 59, 233 (2008).

[72] D. Segal and B. K. Agarwalla, Annual Review of Physical Chemistry 67, 185 (2016).

[73] M. Kobus, P. H. Nguyen, and G. Stock, The Journal of Chemical Physics 134, 124518 (2011).

[74] A. Arkhipov and G. Kuperberg, Geometry \& Topology Monographs 18, 1 (2012).

[75] S. Woutersen and H. J. Bakker, Nature 402, 507 (1999).

[76] J. Lindner, P. Vhringer, M. S. Pshenichnikov, D. Cringus, D. A. Wiersma, and M. Mostovoy, Chemical Physics Letters 421, 329 (2006).

[77] K. Ramasesha, L. De Marco, A. Mandal, and A. Tokmakoff, Nat Chem 5, 935 (2013).

[78] R. A. Horn and C. R. Johnson, Topics in Matrix Analysis (Cambridge University Press, 1991).

[79] R. D. Amos, N. C. Handy, W. H. Green, D. Jayatilaka, A. Willetts, and P. Palmieri, The Journal of Chemical Physics 95, 8323 (1991).

[80] W. Mizukami and D. P. Tew, The Journal of Chemical Physics 139, 194108 (2013).

[81] G. Kirchmair, B. Vlastakis, Z. Leghtas, S. E. Nigg, H. Paik, E. Ginossar, M. Mirrahimi, L. Frunzio, S. M. Girvin, and R. J. Schoelkopf, Nature 495, 205 (2013).

[82] I. Shomroni, S. Rosenblum, Y. Lovsky, O. Bechler, G. Guendelman, and B. Dayan, Science 345, 903 (2014).

[83] L. De Santis, C. Antón, B. Reznychenko, N. Somaschi, G. Coppola, J. Senellart, C. Gómez, A. Lemaître, I. Sagnes, A. G. White, L. Lanco, A. Auffèves, and P. Senellart, Nat Nano 12, 663 (2017).

[84] M. A. Nielsen and I. L. Chuang, Quantum computation and quantum information (Cambridge University Press, Cambridge, 2000).

[85] C. Lacava, M. J. Strain, P. Minzioni, I. Cristiani, and M. Sorel, Opt. Express 21, 21587 (2013).

[86] C. Lacava, S. Stankovic, A. Z. Khokhar, T. D. Bucio, F. Y. Gardes, G. T. Reed, D. J. Richardson, and P. Petropoulos, Scientific Reports 7, 22 (2017).

[87] N. Matsuda, R. Shimizu, Y. Mitsumori, H. Kosaka, and K. Edamatsu, Nat Photon 3, 95 (2009).

[88] V. Venkataraman, K. Saha, and A. L. Gaeta, Nat Photon 7, 138 (2013).

[89] A. Feizpour, M. Hallaji, G. Dmochowski, and A. M. Steinberg, Nat Phys 11, 905 (2015).

[90] H. Choi, M. Heuck, and D. Englund, Phys. Rev. Lett. 
118, 223605 (2017)

[91] E. Knill, R. Laflamme, and G. Milburn, Nature 409, 46 (2001).

[92] J. Huh, G. G. Guerreschi, B. Peropadre, J. R. McClean, and A. Aspuru-Guzik, Nat Photon 9, 615 (2015).

[93] A. Arkhipov, Phys. Rev. A 92, 062326 (2015).

[94] N. J. Russell, L. Chakhmakhchyan, J. L. OBrien, and A. Laing, New Journal of Physics 19, 033007 (2017).

[95] J. B. Spring, P. L. Mennea, B. J. Metcalf, P. C. Humphreys, J. C. Gates, H. L. Rogers, C. Söller, B. J. Smith, W. S. Kolthammer, P. G. R. Smith, and I. A. Walmsley, Optica 4, 90 (2017).

[96] H. Wang, Z.-C. Duan, Y.-H. Li, S. Chen, J.-P. Li, Y.-M. He, M.-C. Chen, Y. He, X. Ding, C.-Z. Peng, C. Schneider, M. Kamp, S. Höfling, C.-Y. Lu, and J.-W. Pan, Phys. Rev. Lett. 116, 213601 (2016).
[97] H. Lee, T. Chen, J. Li, O. Painter, and K. J. Vahala, Nat. Comms. 3, 867 (2012).

[98] O. Christiansen, H. Koch, and P. Jargensen, Chemical Physics Letters 243, 409 (1995).

[99] F. Weigend and R. Ahlrichs, Phys. Chem. Chem. Phys. 7, 3297 (2005).

[100] A. Khn and C. Httig, The Journal of Chemical Physics 119, 5021 (2003).

[101] S. Scheel, K. Nemoto, W. J. Munro, and P. L. Knight, Phys. Rev. A 68, 032310 (2003).

[102] D. Kraft, A Software Package for Sequential Quadratic Programming, Tech. Rep. (DFVLR-FB 88-28, 1988).

[103] E. Jones, T. Oliphant, P. Peterson, et al., "SciPy: Open source scientific tools for Python," (2001-).

[104] J. Eisert, Phys. Rev. Lett. 95, 040502 (2005).

[105] J. A. Nelder and R. Mead, Computer Journal 7, 308 (1965). 


\section{Appendix A: Quantum Chemistry details}

The electronic ground state of $\mathrm{H}_{2} \mathrm{CS}, \mathrm{SO}_{3}, \mathrm{HNCO}, \mathrm{HFHF}, \mathrm{N}_{4}, \mathrm{P}_{4}, \mathrm{~N}$-methylacetamide, $\mathrm{H}_{2} \mathrm{O}$ and $\mathrm{NH}_{3}$ are all closed-shell singlet states. For $\mathrm{H}_{2} \mathrm{CS}, \mathrm{SO}_{3}, \mathrm{HNCO}, \mathrm{HFHF}, \mathrm{N}_{4}, \mathrm{P}_{4}$ and $\mathrm{NH}_{3}$, the equilibrium geometries and force constant matrices were computed using $\mathrm{CC} 2$, an approximate coupled-cluster correlated wavefunction method, and the TVZPP atomic orbital basis sets [65, 98, 99]. For N-methylacetamide, density functional theory with the B3LYP functional and a split-valence atomic orbital basis set was used. The planar equilibrium geometry and force constant matrix of the first electronic excited state of $\mathrm{NH}_{3}$ were computed at the CC2/TZVPP level of theory, where the Hessian was computed by finite difference using the nuclear gradients of the excited electronic state [100]. For the $\mathrm{H}_{2} \mathrm{O}$ molecule, the equilibrium geometry, second, third and semi-diagonal quartic force constant matrices were taken from reference [80]. The normal modes and harmonic wavenumbers for each molecule and electronic state were computed by diagonalising the projected mass-weighted force constant matrix.

Since the geometries of $\mathrm{NH}_{3}$ in its ground and excited states differ, the vector space spanned by the normal modes differ. The unitary transformation $U_{\mathrm{GE}}$ used to approximately model the projection of the vibrational modes of the ground state $V_{G}$ to those of the excited state $V_{E}$ is the one that obtains maximal overlap in the orthogonal procrustes sense

$$
U_{\mathrm{GE}}=\min _{U}\left\|V_{E}-V_{G} U\right\|_{2}
$$

\section{Appendix B: Experimental details}

\section{Photon source}

The photonic states used as input for the simulations are generated using 140 fs pulses of light produced by a Ti:Sapphire laser with emission wavelength centred at $808 \mathrm{~nm}$ and a repetition rate of $80 \mathrm{MHz}$. Frequency doubling of these pulses is achieved with a Barium Borate (BBO) nonlinear crystal that is followed by dichroic mirrors used to separate the light at $404 \mathrm{~nm}$ from the pump beam. The $404 \mathrm{~nm}$ pulses are then used to induce spontaneous parametric down conversion (SPDC) in a Bismuth Borate (BiBO) nonlinear crystal that generates the pairs of single photons used as input for the experiment. A $3 \mathrm{~nm}$ bandwidth interferometric filter centred at $808 \mathrm{~nm}$ allows for the collection of photons generated at the same wavelength by the SPDC process. Up to two pairs of photons generated by the SPDC process are collected from four points of the emission cone acting as two independent photon-pair sources. Four polarisation maintaining fibres route these photons either to the integrated photonic chip or directly to detectors to herald the generation of the multiphoton input state.

When either source is connected directly to the detectors, the ratio between the coincidence detection rate and the single detection rate is $12 \%$. Taking into account the detector efficiency, this translates to a heralding efficiency of around $24 \%$.

The indistinguishability, measured by Hong-Ou-Mandel dip visibility, for photons generated from the same pair source is $\sim 0.98$ while for photons generated in two different pair sources, the HOM visibility is $\sim 0.91$.

\section{Integrated photonic chip}

The integrated planar lightwave circuit interferometer [56] consists of an array of six germanium doped silica cores surrounded by a silica cladding operating as single mode waveguides for the working wavelength. This device is designed to perform any unitary transformation of the optical modes corresponding to the integrated waveguides using the theoretical scheme described in Ref [67]. This scheme requires 15 beamsplitters with adjustable splitting ratio and 15 variable phase shifters, which are implemented using 30 integrated directional couplers with 50:50 splitting ratio and 30 thermal phase shifters. Each of the thermal phase shifters consists of a metallic electrical heater that can be independently fed with current up to $100 \mathrm{~mA}$ supplied by an electronic circuit externally controlled via computer. Two optical fibre arrays are coupled and glued at the input and output ports ensuring coupling stability. Further details of this reconfigurable device can be found in [56].

\section{Detectors}

The photonic output state is measured by means of an array of single photon detectors. The experimental setup is equipped with 12 silicon single-photon avalanche photodiode detectors with an average efficiency of 50\% connected 
to a coincidence counting card time-tagging 16 simultaneous channels in a time window usually set to be around 2 ns during these experiments. For each channel it is possible to set a specific time delay that is used by the counting card to compensate the discrepancy in the signals arrival time introduced in the experiment by optical fibres, detectors, electronics and coaxial cables.

\section{Input state configurations}

All experiments are performed using one or both of the SPDC sources described above. Different input Fock states are prepared with a combination of heralding signals and postselection.

- Single photon input states, $|1\rangle$, are obtained using a single pair source and measuring coincidental detection between one of the photons routed through the chip and the second photon connected directly to a detector as a herald.

- Two photon input states of the form $|2\rangle$ are obtained by routing a single arm of a pair source through the chip and postselecting upon coincidental detection of two photons at the output.

- Two photon input terms of the form $|1\rangle|1\rangle$ are obtained using a single pair source and routing both the photons through the chip, postselecting upon coincidental detection of two photons at the output.

- Four photon input terms of the form $|2\rangle|2\rangle$ are obtained using the same configuration and postselecting upon coincidental detection of four photons at the output.

- Three photon input states of the form $|1\rangle|1\rangle|1\rangle$ required both pair sources to be employed with three of the fibres connected to the chip and the fourth directly to a detector as a herald. The postselection is based on the detection of the heralding photon and of three photons at the output of the chip.

- The three photon input $|2\rangle|1\rangle$ required for the simulation of anharmonic evolution of $\mathrm{H}_{2} \mathrm{O}$ is obtained via the same configuration as the above $|2\rangle|2\rangle$ input, and using the chip to implement a beamsplitter operation between one of the input modes and two of the output modes. One of these output modes is then used as a heralding signal for the $|2\rangle|1\rangle$ input. Further heralding is then required to confirm the correct operation of the NPS gate.

\section{Experimental procedure}

The procedure for collecting experimental data begins with a set of experiments to measure detector efficiencies. A heralded single photon is input to the chip which is set to route the photon to each output mode. Collecting detection events for a fixed period of time for each output mode then allows us to estimate the relative detector efficiencies by dividing all the count rates by the largest count rate. The input state is then set to one of the configurations described above and the experiment is performed by logging all coincidental detections at the output with the integrated chip set to perform the set of unitaries required for the simulation. These experimental counts are then corrected using the measured efficiencies by dividing the total counts in a detection pattern by the product of the relative detector

efficiencies of the detectors in that pattern. These corrected counts are then used to estimate the expectation value of projecting the output state onto a detection pattern as the frequency of corrected counts in that pattern to the total number of corrected counts.

Finally, the statistical noise in the estimates of these probabilities resulting from the finite counting statistics is estimated via a bootstrapping approach, where 1000 sets of data are resampled from Poissonian distributions with means given by the experimentally measured counts, and the standard deviation of the probabilities estimated from these sets are used to provide error bars on plots.

\section{Probabilistic number resolving}

Many of the experiments reported in this article require the ability to resolve multiple occupancy in the output modes. Although number-resolving detectors were not available in this experiment we are able to circumvent this requirement by performing probabilistic number resolving between one and two photons using auxiliary fibre beamsplitters (FBS) and detectors. This is achieved by inserting a FBS at each of the relevant output fibres and connecting both output modes to detectors; when there are two photons incident at the FBS there is then a probability that both the detectors generate a signal. For a beamsplitter with reflectivity $r$ connected to detectors with efficiencies $\eta_{1}$ and $\eta_{2}$ 
, the probability of detecting a coincidental detection given a two-photon input is $2 r(1-r) \eta_{1} \eta_{2}$. Performing detector efficiency measurements as described in the previous section, the count rates in each detector will be proportional to their effective efficiencies, $r \eta_{1}$ and $(1-r) \eta_{2}$. As before, relative efficiencies are then calculated by dividing by the maximum count rate and all coincidence counts are re-normalised by the product of these relative effective efficiencies. A final correction is then applied to account for multiple occupancy, for instance in a two photon experiment counts corresponding to the same output mode are halved, whereas counts corresponding to different output modes are averaged over the four possible ways of detecting such an output.

\section{Appendix C: Molecule details}

Here we include all computed vibrational frequencies and local basis transformations used in the work.

\section{1. $\mathrm{H}_{2} \mathrm{CS}$}

The frequencies of the normal modes are calculated to be

$$
\{3195.45,3102.74,1501.77,1061.45,1017.08,1008.04\} \mathrm{cm}^{-1}
$$

and the transformation between the normal modes and localised modes is given by

$$
U_{L}=\left(\begin{array}{cccccc}
0.563 & 0.572 & 0.332 & 0.251 & 0 . & 0.427 \\
-0.563 & 0.572 & 0.332 & 0.251 & 0 . & -0.427 \\
-0.427 & -0.392 & 0.572 & 0.138 & 0 . & 0.564 \\
0 . & -0.196 & -0.355 & 0.914 & 0 . & 0 . \\
0 . & 0 . & 0 . & 0 . & 1 . & 0 . \\
-0.427 & 0.392 & -0.572 & -0.138 & 0 . & 0.564
\end{array}\right)
$$

\section{HFHF}

The frequencies of the normal modes are calculated to be

$$
\{4068.29,3981.51,578.25,464.46,207.95,148.93\} \mathrm{cm}^{-1}
$$

and the transformation between the normal modes and localised modes is given by

$$
U_{L}=\left(\begin{array}{cccccc}
0.768 & 0.147 & 0.281 & 0 . & -0.410 & -0.377 \\
-0.178 & 0.920 & 0.265 & 0 . & 0.220 & -0.046 \\
0.064 & -0.303 & 0.844 & 0 . & 0.357 & 0.254 \\
0 . & 0 . & 0 . & 1 . & 0 . & 0 . \\
0.607 & 0.135 & -0.351 & 0 . & 0.462 & 0.525 \\
-0.076 & 0.145 & 0.123 & 0 . & -0.665 & 0.718
\end{array}\right)
$$

\section{3. $\mathrm{HNCO}$}

The frequencies of the normal modes are calculated to be

$$
\{3694.87,2260.22,1241.85,805.38,605.69,552.52\} \mathrm{cm}^{-1}
$$

and the transformation between the normal modes and localised modes is given by

$$
U_{L}=\left(\begin{array}{cccccc}
0.826 & 0.116 & -0.198 & -0.438 & 0 . & 0.272 \\
-0.066 & 0.982 & 0.120 & 0.001 & 0 . & -0.129 \\
0.157 & -0.090 & 0.971 & -0.085 & 0 . & 0.133 \\
0.537 & -0.037 & 0.039 & 0.667 & 0 . & -0.514 \\
0 . & 0 . & 0 . & 0 . & 1 . & 0 . \\
0.028 & 0.111 & -0.050 & 0.597 & 0 . & 0.792
\end{array}\right)
$$




\section{4. $\mathrm{N}_{4}$}

The frequencies of the normal modes are calculated to be

$$
\{1366.42,1015.85,947.85,916.73,412.35,366.86\} \mathrm{cm}^{-1}
$$

and the transformation between the normal modes and localised modes is given by

$$
U_{L}=\left(\begin{array}{cccccc}
0.191 & -0.356 & -0.611 & 0.494 & 0 . & 0.469 \\
0.658 & 0.611 & -0.356 & -0.011 & 0 . & -0.258 \\
0.658 & -0.611 & 0.356 & -0.011 & 0 . & -0.258 \\
0.191 & 0.356 & 0.611 & 0.494 & 0 . & 0.469 \\
0 . & 0 . & 0 . & 0 . & 1 . & 0 . \\
0.244 & 0 . & 0 . & -0.716 & 0 . & 0.654
\end{array}\right)
$$

\section{5. $\mathbf{P}_{4}$}

The frequencies of the normal modes are calculated to be

$$
\{608.25,460.20,460.20,460.20,366.27,366.25\} \mathrm{cm}^{-1}
$$

and the transformation between the normal modes and localised modes is given by

$$
U_{L}=\left(\begin{array}{cccccc}
0.5 & -0.676 & -0.096 & -0.533 & 0 . & 0 . \\
0.5 & 0.727 & 0.074 & -0.465 & 0 . & 0 . \\
0 . & 0 . & 0 . & 0 . & 0.853 & -0.522 \\
0.5 & 0.059 & -0.691 & 0.519 & 0 . & 0 . \\
-0.5 & 0.110 & -0.713 & -0.480 & 0 . & 0 . \\
0 . & 0 . & 0 . & 0 . & 0.522 & 0.853
\end{array}\right)
$$

\section{6. $\mathrm{SO}_{3}$}

The frequencies of the normal modes are calculated to be

$$
\{1334.97,1334.55,966.96,497.57,497.52,469.50\} \mathrm{cm}^{-1}
$$

and the transformation between the normal modes and localised modes is given by

$$
U_{L}=\left(\begin{array}{cccccc}
0.608 & -0.346 & -0.577 & -0.218 & 0.362 & 0 . \\
0 . & 0.701 & -0.579 & 0.417 & 0 . & 0 . \\
0.608 & 0.346 & 0.577 & 0.218 & 0.362 & 0 . \\
0 . & -0.519 & -0.012 & 0.855 & 0 . & 0 . \\
-0.511 & 0 . & 0 . & 0 . & 0.859 & 0 . \\
0 . & 0 . & 0 . & 0 . & 0 . & 1 .
\end{array}\right)
$$

\section{NMA}

The vibrational frequencies of NMA are calculated to be

$$
\begin{aligned}
& \{3589.68,3143.77,3113.38,3111.68,3060.23,3024.31,3000.1,1808.96,1572.7,1484.02,1481.06,1469.21, \\
& 1452.61,1427.76,1391.94,1279.31,1170.4,1150.05,1120.42,1053.5,995.522,876.075,637.311,628.305, \\
& 475.592,436.109,291.874,176.452,96.9364,39.7879\} \mathrm{cm}^{-1}
\end{aligned}
$$

and the transformation used is $U_{L} \oplus \mathbb{1}_{24}$ where we select the subset

$$
\{1279.31,1170.4,1120.42,995.522,876.075,628.305\}
$$


to be the first six modes.

$$
U_{L}=\left(\begin{array}{cccccc}
0.796 & -0.007 & -0.401 & -0.345 & 0.050 & -0.290 \\
-0.115 & 0.794 & 0.141 & -0.178 & 0.500 & -0.233 \\
0.518 & -0.085 & 0.807 & 0.139 & 0.156 & 0.172 \\
0.211 & 0.147 & -0.312 & 0.889 & 0.214 & -0.012 \\
-0.190 & -0.583 & 0.006 & -0.031 & 0.704 & -0.357 \\
0.059 & -0.011 & -0.267 & -0.197 & 0.427 & 0.839
\end{array}\right)
$$

\section{8. $\mathrm{H}_{2} \mathrm{O}$}

The vibrational frequencies of the normal modes in the harmonic model are

$$
\{3914.92,3787.59,1627.01\} \mathrm{cm}^{-1}
$$

and the transformation between normal and local modes is

$$
U_{L}=\left(\begin{array}{ccc}
0.70711 & -0.70709 & -0.00502 \\
0.70711 & 0.70709 & 0.00502 \\
0 . & -0.00710 & 0.99997
\end{array}\right)
$$

Now, restricting to the subspace of the asymmetric and symmetric stretch modes and adjusting the energy levels for anharmonic potentials via perturbation theory results in single excitation energy levels of

$$
\{3740.05,3619.68\} \mathrm{cm}^{-1} \text {. }
$$

With two excitations the $\{20,02,11\}$ energy levels become

$$
\{7391.43,7154.35,7206.46\} \mathrm{cm}^{-1}
$$

and the normal to local transformation for this subspace is given by

$$
U_{L}=\left(\begin{array}{ccc}
0.70711 & -0.70711 & 0 . \\
0.70711 & 0.70711 & 0 . \\
0 . & 0 . & 1 .
\end{array}\right)
$$

\section{9. $\mathbf{N H}_{3}$}

The vibrational frequencies of the ground electronic state are given by

$$
\{3646.13,3646.09,3503.94,1678.57,1678.4,1065.59\} \mathrm{cm}^{-1}
$$

and the frequencies of the excited state are

$$
\{2590.27,2452.95,2451.97,1052.62,1050.93,750.124\} \mathrm{cm}^{-1} \text {. }
$$

The transformation between the two sets of modes is given by

$$
U_{\mathrm{GE}}=\left(\begin{array}{cccccc}
-0.085 & -0.289 & -0.216 & 0.090 & 0.913 & -0.147 \\
-0.058 & -0.011 & 0.096 & 0.023 & 0.169 & 0.979 \\
0.935 & -0.023 & -0.341 & -0.031 & 0.017 & 0.087 \\
0.131 & 0.859 & 0.313 & -0.118 & 0.358 & -0.072 \\
0.308 & -0.336 & 0.819 & 0.331 & 0.0694 & -0.0856 \\
-0.052 & 0.256 & -0.244 & 0.931 & -0.071 & 0.014
\end{array}\right)
$$

and the transformation between normal and local coordinates in the excited state is given by

$$
U_{L}=\left(\begin{array}{cccccc}
0.575 & -0.542 & -0.576 & -0.058 & 0.200 & 0 . \\
0.579 & 0.767 & -0.185 & 0.195 & -0.062 & 0 . \\
0.578 & -0.223 & 0.757 & -0.163 & -0.131 & 0 . \\
0.012 & -0.139 & 0.212 & 0.857 & 0.448 & 0 . \\
-0.010 & 0.221 & 0.126 & -0.444 & 0.859 & 0 . \\
0 . & 0 . & 0 . & 0 . & 0 . & 1 .
\end{array}\right)
$$




\section{Appendix D: Simulating open system dynamics}

\section{Dephasing}

To simulate uniform dephasing across six vibrational modes we consider the Lindblad master equation

$$
\dot{\rho}=\mathcal{L}=-i[\hat{H}, \rho]+\frac{\Gamma}{6} \sum_{k=1}^{5}\left(\hat{Z}(k) \rho \hat{Z}^{\dagger}(k)-\frac{1}{2}\left\{\hat{Z}^{\dagger}(k) \hat{Z}(k), \rho\right\}\right)
$$

where $\hat{Z}(k)=\sum_{j} \exp (i 2 \pi j k / 6) a_{j}^{\dagger} a_{j}$, i.e. $Z(k)$ are six dimensional Heisenberg-Weyl matrices. Solving this equation results in the channel $\rho(t)=\sum_{k} M_{k}(t) e^{-i \hat{H} t / \hbar} \rho(0) e^{i \hat{H} t / \hbar} M_{k}^{\dagger}(t)$ with time-dependent Kraus operators

$$
\begin{aligned}
M_{0}(t) & =\sqrt{\frac{1+5 e^{-\Gamma t}}{6}} \sum_{j=1}^{6} a_{j}^{\dagger} a_{j} \\
M_{k>0}(t) & =\sqrt{\frac{1-e^{-\Gamma t}}{6}} \sum_{j=1}^{6} \exp \{i 2 \pi j k / 6\} a_{j}^{\dagger} a_{j}
\end{aligned}
$$

where $\Gamma^{-1}$ is usually referred to as the $T_{2}$ time. Including the change of basis to the local vibrational modes, we can therefore simulate these dephasing dynamics for a set of vibrational modes with arbitrary $T_{2}$ times by statistical averaging the expectation values obtained from experiments implementing the transfer matrices $U(t, j)=U_{L} Z(j) e^{-i H t / \hbar} U_{L}^{\dagger}$. In the case of the NMA molecule data plotted in Fig. 2, we use $T_{2}=0.53 p s$.

\section{Appendix E: Thermalisation}

To simulate vibrational relaxation for a single excitation we consider the Lindblad master equation

$$
\begin{aligned}
\dot{\rho}=\mathcal{L}(\rho)= & -i[\hat{H}, \rho]+\sum_{j=1,2} \Gamma_{1}\left(|3\rangle\langle j|\rho| j\rangle\langle 3|-\frac{1}{2}\{|j\rangle\langle j|, \rho\}\right)+\Gamma_{2}\left(|0\rangle\langle 3|\rho| 3\rangle\langle 0|-\frac{1}{2}\{|3\rangle\langle 3|, \rho\}\right) \\
& +\sum_{j=1,2} \Gamma_{3}\left(|0\rangle\langle j|\rho| j\rangle\langle 0|-\frac{1}{2}\{|j\rangle\langle j|, \rho\}\right)
\end{aligned}
$$

Where $|j\rangle \equiv a_{j}^{\dagger} \mid$ vac $\rangle$ and $|0\rangle \equiv \mid$ vac $\rangle$. Solving Eq. E1 in this case results in time dependent Kraus operators

$$
\begin{aligned}
& M_{1}(t)=\sqrt{1-e^{-\Gamma_{1} t}}|3\rangle\langle 1| \\
& M_{2}(t)=\sqrt{1-e^{-\Gamma_{1} t}}|3\rangle\langle 2| \\
& M_{3}(t)=\sqrt{1-e^{-\Gamma_{2} t}}|0\rangle\langle 3| \\
& M_{4}(t)=\sqrt{1-e^{-\Gamma_{3} t}}|0\rangle\langle 1| \\
& M_{5}(t)=\sqrt{1-e^{-\Gamma_{3} t}}|0\rangle\langle 2| \\
& M_{0}(t)=\sqrt{\mathbb{1}-\sum_{i=1}^{5} M_{i}^{\dagger}(t) M_{i}(t)}
\end{aligned}
$$

The evolution between the non-vacuum states can then be simulated by the set of transfer matrices $K$

$$
K_{i<3}(t)=U_{L} M_{i}(t) e^{-i H t / \hbar} U_{L}^{\dagger}
$$

This case cannot be simulated as a convex sum of unitary evolutions. However, we can implement general linear transformations using the concept of unitary dilation at the cost of a non-unit probability of success. Any $m \times m$ matrix $A$ with spectral norm $\|A\|_{2} \leq 1$ can be embedded as the top-left block of a $2 m \times 2 m$ unitary matrix $U_{A}$. All possible unitary dilations of A can be parameterised using the Cosine-Sine decomposition [78]. By considering a singular value decomposition of $A$

$$
A=U \cos (\theta) V^{\dagger}
$$


where $\cos (\theta)=\operatorname{diag}\left(\cos \theta_{1}, \ldots, \cos \theta_{m}\right)$ we can express the possible dilations of $A$ into $2 m$ modes as

$$
U_{A}=\left(\begin{array}{cc}
U & 0 \\
0 & X
\end{array}\right)\left(\begin{array}{cc}
\cos (\theta) & -\sin (\theta) \\
\sin (\theta) & \cos (\theta)
\end{array}\right)\left(\begin{array}{cc}
V^{\dagger} & 0 \\
0 & Y^{\dagger}
\end{array}\right)
$$

where $X, Y \in U(m)$. In this experiment we used $X=Y=\mathbb{1}$. Using this technique, we are then able to simulate each of the set of transformations $K$ using the dilations $U_{K}$.

The terms involving $M_{3-5}$ can be simulated via introducing relative losses to the modes. This could be achieved by coupling to further ancilla modes and this time a detection in these modes would correspond to the projection $|0\rangle\langle 0|$. Here instead, we simply apply a time-dependent effective efficiency to the detected counts on each mode. Finally, $M_{0}(t)$ is diagonal and commutes with $U_{L}$, meaning that it can also be achieved via a rescaling of the detected counts from the ideal unitary evolution $U_{L} e^{-i H t / \hbar} U_{L}^{\dagger}$.

Statistically mixing these experiments results in the correct expectation values and states $\rho(t)$ for the thermalisation process. We used the $\omega_{i}$ from the previous section and $\Gamma_{1}=0.24 \mathrm{ps}, \Gamma_{2}=0.26 \mathrm{ps}$ and $\Gamma_{3}=1.36 \mathrm{ps}$ taken from experimental data in $[76]$.

\section{Appendix F: NPS gates}

In order to implement the NPS gates, we wish to perform diagonal unitaries in the Fock basis characterised by the evolution

$$
a_{0}|0\rangle+a_{1}|1\rangle+a_{2}|2\rangle \rightarrow a_{0}|0\rangle+a_{1} e^{i \phi_{1}}|1\rangle+a_{2} e^{i \phi_{2}}|2\rangle
$$

for a single optical mode. We will search for gates which use a single ancilla photon, injected in an ancilla mode. In this case it is possible to then restrict the search to arbitrary $2 \times 2$ transfer matrices $M$ which can be extended to unitaries using the principle of dilation explained in the previous section. By considering the equations that must be satisfied

$$
\begin{aligned}
\operatorname{Per}\left[M_{(0,1),(0,1)}\right] & =e^{-i \phi_{1}} \operatorname{Per}\left[M_{(1,1),(1,1)}\right] \\
2 \operatorname{Per}\left[M_{(0,1),(0,1)}\right] & =e^{-i \phi_{2}} \operatorname{Per}\left[M_{(2,1),(2,1)}\right]
\end{aligned}
$$

the solutions can be parameterised as

$$
M=\left(\begin{array}{cc}
1-\sqrt{1-e^{i \rho}} & x \\
\frac{y}{x} \sqrt{1-e^{i \rho}} & y
\end{array}\right)\left(\begin{array}{cc}
e^{i \phi_{1}} & 0 \\
0 & 1
\end{array}\right)
$$

where $\rho=\phi_{2}-2 \phi_{1}$. It was proven in Ref. [101] that solutions exist for all choices of $\left\{\phi_{1}, \phi_{2}\right\}$. Optimal gates can then be found by performing the optimisation

$$
\begin{aligned}
& \operatorname{maximise} P_{\text {succ }} \\
& \text { subject to }\|M\|_{2} \leq 1
\end{aligned}
$$

where $P_{\text {succ }}=|y|^{2}$ is the probability that the gate succeeds in performing the desired transformation. The optimisation is performed by using the sequential least squares programming (SLSQP) [102] algorithm included in the SciPy [103] python package.

Fig. 5 shows the success probabilities for the optimised gates for pairs of phases $\left\{\phi_{1}, \phi_{2}\right\}$. Beyond the trivial case of $\left\{\phi_{1}, 2 \phi_{1}\right\}$, where $P_{\text {succ }}=1$, globally optimal solutions occur for $\left\{\phi_{1}, \phi_{1}+\pi\right\}$ where $P_{\text {succ }}=1 / 4$. These solutions can be seen to be generalisations of the KLM NS gate. For the set of worst case $\left\{\phi_{1}, \phi_{2}\right\}$ pairs, we find that $P_{\text {succ }}=1 / 6$.

Ref. [104] establishes an upper bound on the probability of success of NPS gates of the form $\{0, \phi\}$ with arbitrary ancilla states of $P_{\text {succ }} \leq(3-\cos (\pi-\phi))^{2} / 16$. For the case when $\phi=\pi$ this bound is saturated by the KLM NS gate. However, we find that in restricting to such single photon ancillas, this upper bound is not generally achievable. Furthermore, we observed that these probabilities were not improved by considering separable ancilla states containing two photons. We leave it as an open problem to establish whether the upper bound from [104] is achievable and what form of ancilla state is required. 


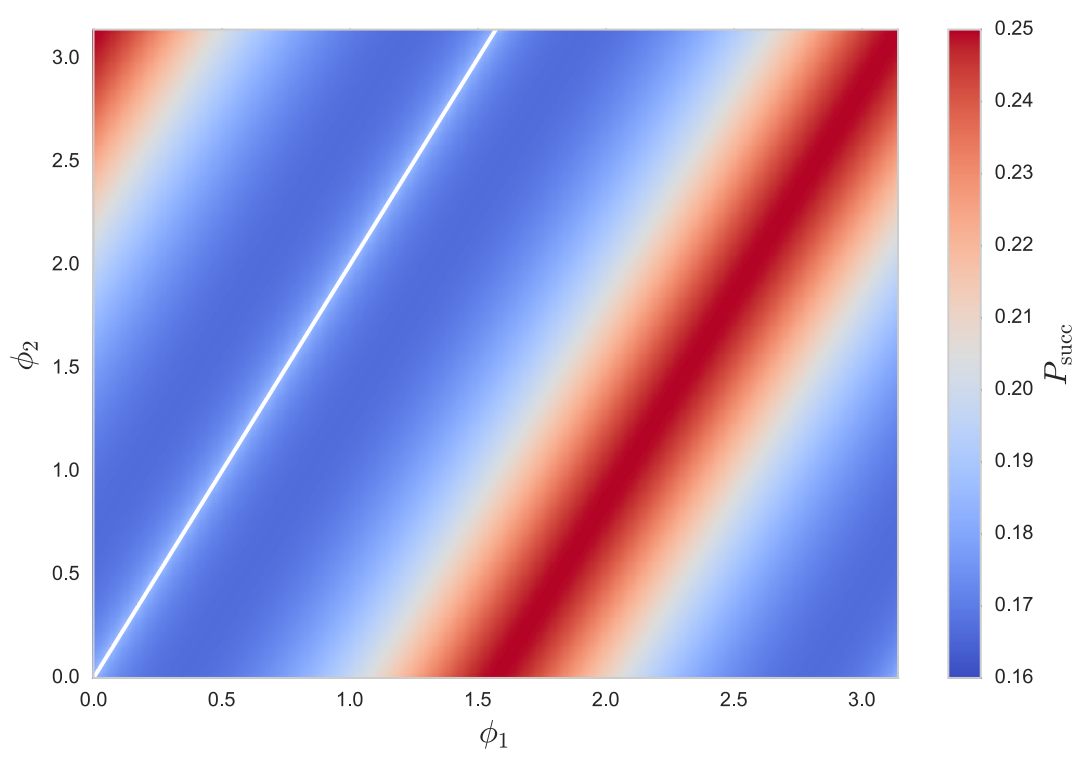

FIG. 5. Optimised success probability $\left(P_{\text {succ }}\right)$ for a nonlinear phase shift (NPS) gate mapping performing the transformation $a_{0}|0\rangle+a_{1}|1\rangle+a_{2}|2\rangle$ to $a_{0}|0\rangle+a_{1} e^{i \phi_{1}}|1\rangle+a_{2} e^{i \phi_{2}}|2\rangle$ using two ancilla modes and one ancilla photon. The white line is the curve $\phi_{2}=2 \phi_{1}$, along which $P_{\text {succ }}=1$.

\section{Appendix G: AFC Algorithm}

In the adaptive feedback algorithm experiment we begin with an initial state of two photons in orthogonal optical modes. We then imagine we are able to prepare a set of states defined by this state subject to a unitary mixing of the vibrational modes, $U(\vec{\theta})$. We ignore the sixth mode as this remains uncoupled from the others (see Molecule details section) and perform an arbitrary unitary transformation upon the remaining five modes. There are 14 free parameters, $\theta_{i}$, in this state preparation, which are mapped to the phase shifts and beamsplitter splitting ratios within a linear optical network. These are the parameters which are optimised in the experiment. This parameterised initial state is then used as input to a simulation where a vibrational state in the ground state of $\mathrm{NH}_{3}$ is electronically excited, evolves for a time $t$ and we look to maximise the probability of bunching in the modes corresponding to local $\mathrm{NH}$ stretches and minimise all other bunching.

Our feedback algorithm is a type of random restart hill climbing optimisation with an objective function which depends on observed data, which is implemented as follows. We begin with a set of uniformly random parameters. We then collect all two-photon coincidences at the output for a series of time steps separated by $\Delta t$. After correcting for detector efficiencies and the inefficient detection of multiple photon mode-occupancies, the probabilities of bunched events

$$
p_{\text {bunch }}^{i}(\vec{\theta}, t)=\left|\left\langle 2_{i} \mid \psi(\vec{\theta}, t)\right\rangle\right|^{2}
$$

can be estimated by the procedure outlined in previous sections, where

$$
|\psi(\vec{\theta}, t)\rangle=\sum_{j, k} U_{j 1}(\vec{\theta}, t) U_{k 2}(\vec{\theta}, t) a_{j}^{\dagger} a_{k}^{\dagger}|\mathrm{vac}\rangle
$$

and $U(\vec{\theta}, t)=U_{L} e^{-i H t / \hbar} U_{G E}^{\dagger} U(\vec{\theta})$ (see previous sections for definitions of $U_{L}$ and $U_{\mathrm{GE}}$ ). Since we wish to selectively promote dissociation via the three NH stretch modes (corresponding to the first three modes in the localised basis), the quantity we wish to maximise is $p_{1}(t)=\sum_{i=1}^{3} p_{\text {bunch }}^{i}(\vec{\theta}, t)$, whereas we wish to minimise bunching in (and therefore dissociation via) the remaining localised modes $p_{2}(t)=\sum_{i=4}^{6} p_{\text {bunch }}^{i}(\vec{\theta}, t)$. The objective function that we use is

$$
\begin{aligned}
& \min _{\vec{\theta}} C \\
& C=-\alpha \sum_{i} w_{i} \Delta p_{i}
\end{aligned}
$$


with

$$
\begin{aligned}
w_{1} & =1, w_{i>1}=\prod_{j=1}^{i-1}\left(1-\left[p_{1}(j \Delta t)+p_{2}(j \Delta t)\right] / N\right) \\
\Delta p_{i} & =\left[p_{1}(i \Delta t)-p_{2}(i \Delta t)\right] / N \\
\alpha & =\frac{1}{1-\left(\frac{N-1}{N}\right)^{N}}
\end{aligned}
$$

where later times are weighted in order to correct for the possibility of dissociation occurring previously, $N$ is the total number of timesteps and the factor $\alpha$ is used to renormalise the objective function such that it is constrained to the interval $[-1,1]$.

The updating of the values of $\theta_{i}$ is performed using the Nelder-Mead simplex method [105] contained in the SciPy [103] python package. At each iteration, the unitaries are set, data is collected and the objective function $C$ evaluated. The parameters $\vec{\theta}$ are then updated and the procedure is repeated until no further progress is detected, or 2000 evaluations of $C$ have occurred for a given starting set of parameters. The Nelder-Mead method is preferred to gradient based methods in this situation, as the presence of experimental noise is likely to cause $C$ to be nonsmooth.

The optimisation process is repeated for many random initial parameter sets (random restarts) in order to increase the probability of finding the globally optimal solution.

\section{Appendix H: Summary of results}

\begin{tabular}{|c|c|c|c|c|c|}
\hline \multirow{2}{*}{ Molecule } & \multirow{2}{*}{$\begin{array}{l}\text { Figure of } \\
\text { merit }\end{array}$} & \multicolumn{4}{|c|}{ Input state } \\
\hline & & $|1\rangle$ & $|11\rangle$ & $|111\rangle$ & $|22\rangle$ \\
\hline \multirow{2}{*}{$\mathrm{H}_{2} \mathrm{CS}$} & $\overline{\mathcal{F}}$ & $0.999 \pm 0.001$ & $0.992 \pm 0.004$ & $0.971 \pm 0.018$ & $0.969 \pm 0.018$ \\
\hline & $\overline{\mathcal{D}}$ & $0.014 \pm 0.006$ & $0.064 \pm 0.028$ & $0.159 \pm 0.074$ & $0.130 \pm 0.062$ \\
\hline \multirow{2}{*}{$\mathrm{H}_{2} \mathrm{CS}$ (long/short) } & $\overline{\mathcal{F}}$ & $0.999 \pm 0.001$ & $0.995 \pm 0.003$ & - & - \\
\hline & $\overline{\mathcal{D}}$ & $0.024 \pm 0.009$ & $0.063 \pm 0.026$ & - & - \\
\hline \multirow{2}{*}{$\mathrm{HNCO}$} & $\overline{\mathcal{F}}$ & $0.998 \pm 0.001$ & $0.995 \pm 0.002$ & - & - \\
\hline & $\overline{\mathcal{D}}$ & $0.026 \pm 0.009$ & $0.050 \pm 0.015$ & - & - \\
\hline \multirow{2}{*}{$\mathrm{P}_{4}$} & $\overline{\mathcal{F}}$ & $0.999 \pm 0.001$ & $0.996 \pm 0.006$ & - & - \\
\hline & $\overline{\mathcal{D}}$ & $0.025 \pm 0.005$ & $0.067 \pm 0.022$ & - & - \\
\hline \multirow{2}{*}{$\mathrm{N}_{4}$} & $\overline{\mathcal{F}}$ & $0.999 \pm 0.001$ & $0.995 \pm 0.003$ & - & - \\
\hline & $\overline{\mathcal{D}}$ & $0.022 \pm 0.009$ & $0.058 \pm 0.022$ & - & - \\
\hline \multirow{2}{*}{ HFHF } & $\overline{\mathcal{F}}$ & $0.998 \pm 0.001$ & $0.996 \pm 0.002$ & - & - \\
\hline & $\overline{\mathcal{D}}$ & $0.016 \pm 0.005$ & $0.049 \pm 0.023$ & - & - \\
\hline \multirow{2}{*}{$\mathrm{SO}_{3}$} & $\overline{\mathcal{F}}$ & $0.999 \pm 0.001$ & $0.995 \pm 0.00 x$ & - & - \\
\hline & $\overline{\mathcal{D}}$ & $0.022 \pm 0.006$ & $0.070 \pm 0.029$ & - & - \\
\hline \multirow{2}{*}{ NMA } & $\overline{\mathcal{F}}$ & $0.999 \pm 0.001$ & $0.996 \pm 0.003$ & $0.976 \pm 0.018$ & - \\
\hline & $\overline{\mathcal{D}}$ & $0.017 \pm 0.005$ & $0.046 \pm 0.014$ & $0.139 \pm 0.068$ & - \\
\hline \multirow{2}{*}{ NMA (dephased) } & $\overline{\mathcal{F}}$ & $0.998 \pm 0.001$ & - & - & - \\
\hline & $\overline{\mathcal{D}}$ & $0.024 \pm 0.011$ & - & - & - \\
\hline \multirow{2}{*}{$\mathrm{H}_{2} \mathrm{O}$ (thermalisation) } & $\overline{\mathcal{F}}$ & $0.994 \pm 0.004$ & - & - & - \\
\hline & $\overline{\mathcal{D}}$ & $0.024 \pm 0.007$ & - & - & - \\
\hline \multirow{2}{*}{$\mathrm{H}_{2} \mathrm{O}$ (harmonic) } & $\overline{\mathcal{F}}$ & - & $0.998 \pm 0.003$ & - & - \\
\hline & $\overline{\mathcal{D}}$ & - & $0.020 \pm 0.012$ & - & - \\
\hline \multirow{2}{*}{$\mathrm{H}_{2} \mathrm{O}$ (anharmonic) } & $\overline{\mathcal{F}}$ & - & - & - & $0.995 \pm 0.006$ \\
\hline & $\overline{\mathcal{D}}$ & - & - & - & $0.057 \pm 0.024$ \\
\hline $\mathrm{NH}_{3}$ (dissociation) & $C$ & - & -0.886 & - & - \\
\hline
\end{tabular}

Here we include a summary of all data in terms of the distances between theoretical and experimentally estimated distributions. $\mathcal{F}(\mathbf{p}, \mathbf{q})=\sum_{i} \sqrt{p_{i} q_{i}}$ is the statistical fidelity. 

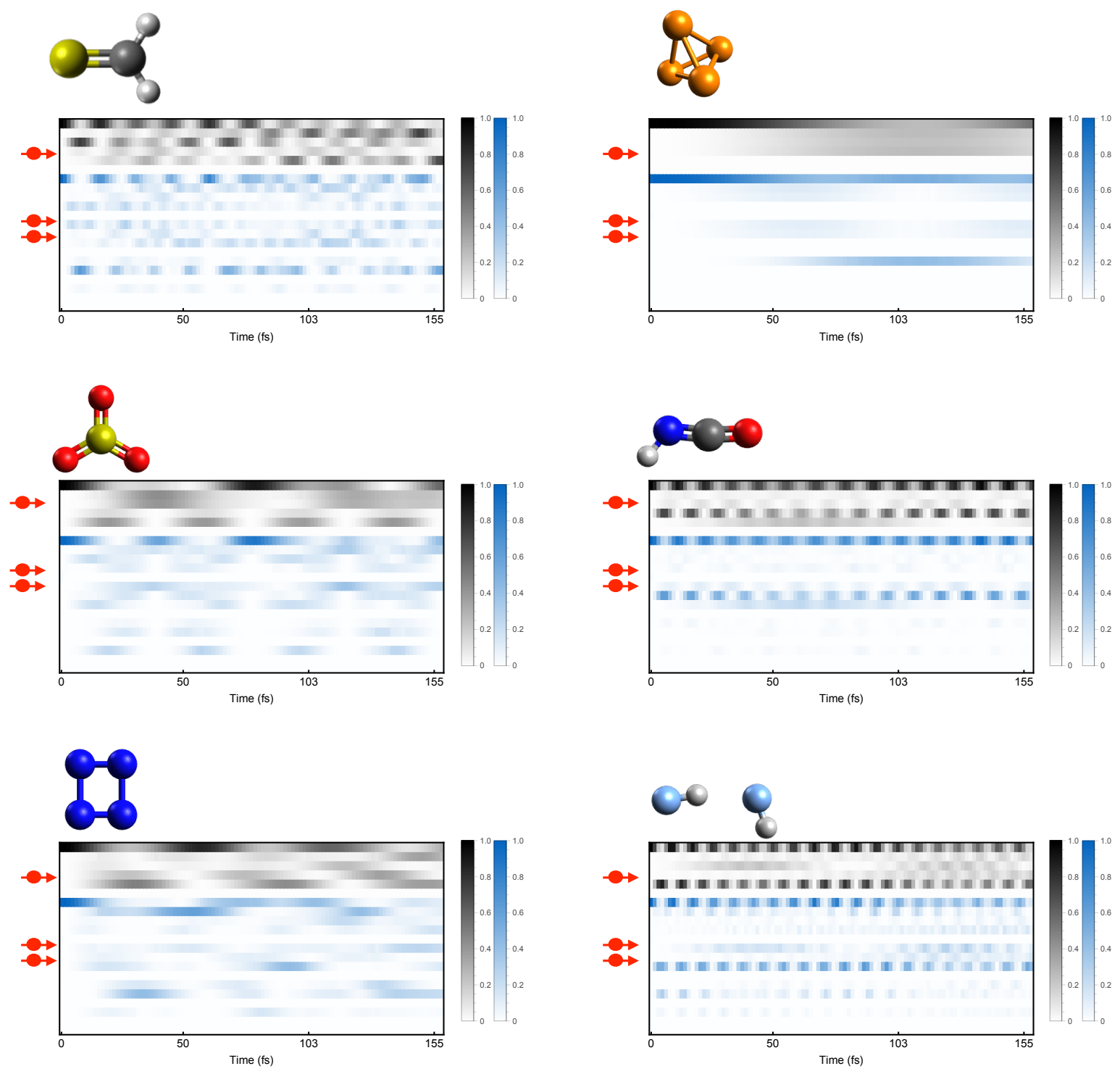

FIG. 6. Four atom molecule data. Full data sets for $|1\rangle$ (black) and $|1\rangle|1\rangle$ (blue) input experiments for $\mathrm{H}_{2} \mathrm{CS}, \mathrm{P}_{4}, \mathrm{SO}_{3}, \mathrm{HNCO}$ $\mathrm{N}_{4}$ and $\mathrm{HFHF}$.

\section{Appendix I: Additional data}

Here we include plots displaying the full data from our experiments. All plots represent the experimentally estimated probabilities for measuring patterns ordered top to bottom as $\{|100000\rangle,|010000\rangle \ldots\}$ for one photon data, $\{|20000\rangle,|020000\rangle \ldots,|110000\rangle,|101000\rangle, \ldots\}$ for two photon data, $\{|111000\rangle,|110100\rangle \ldots\}$ for three photon data and $\{|22000\rangle,|202000\rangle \ldots,|211000\rangle \ldots,|111100\rangle\}$ for four photon data. The mode ordering is defined by the vibrational frequencies defined in the Molecule details section. 


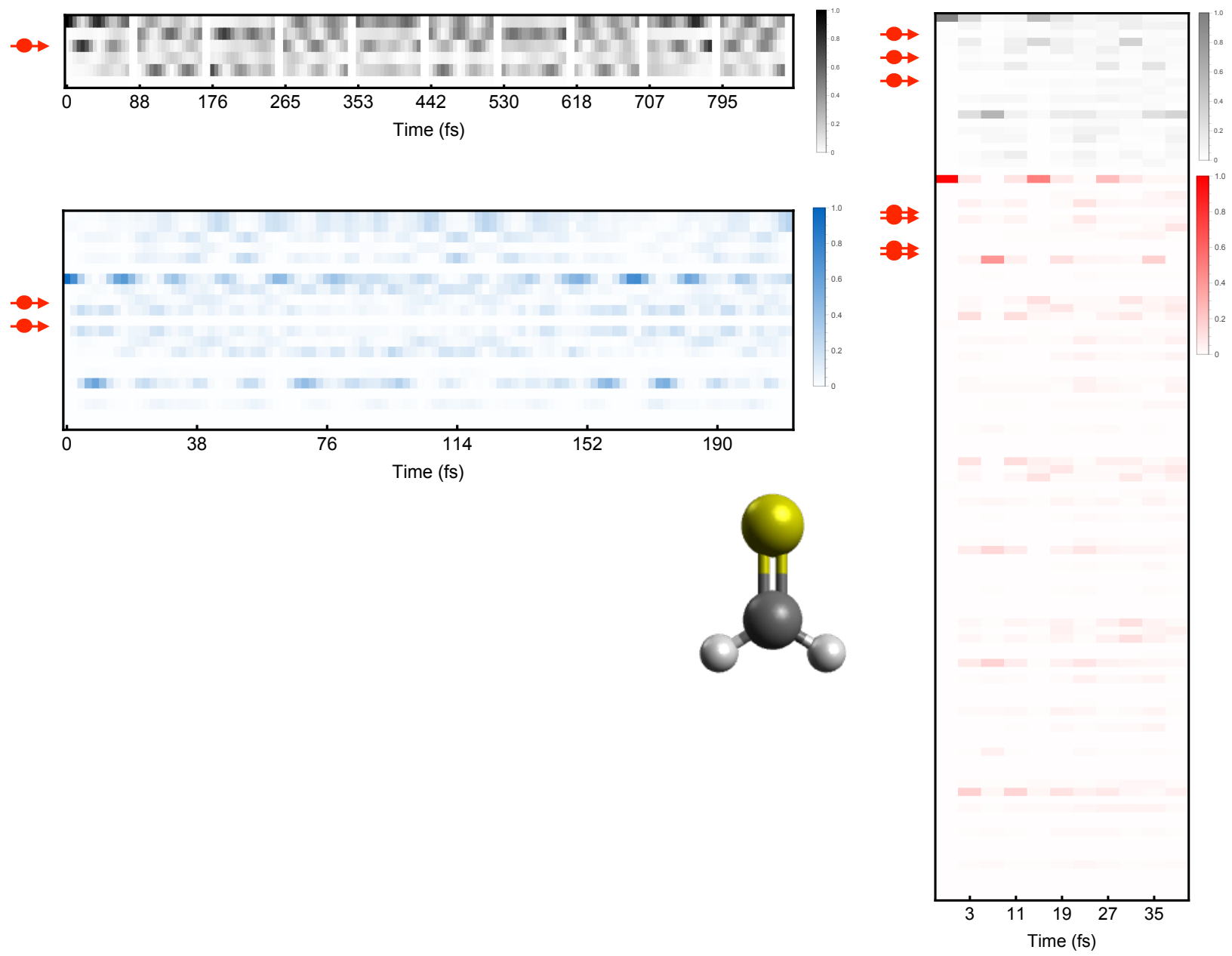

FIG. 7. $\mathrm{H}_{2} \mathrm{CS}$ data. Full data sets for $|1\rangle$ (black), $|1\rangle|1\rangle$ (blue), $|1\rangle|1\rangle|1\rangle$ (grey) and $|2\rangle|2\rangle$ (red) input experiments. 

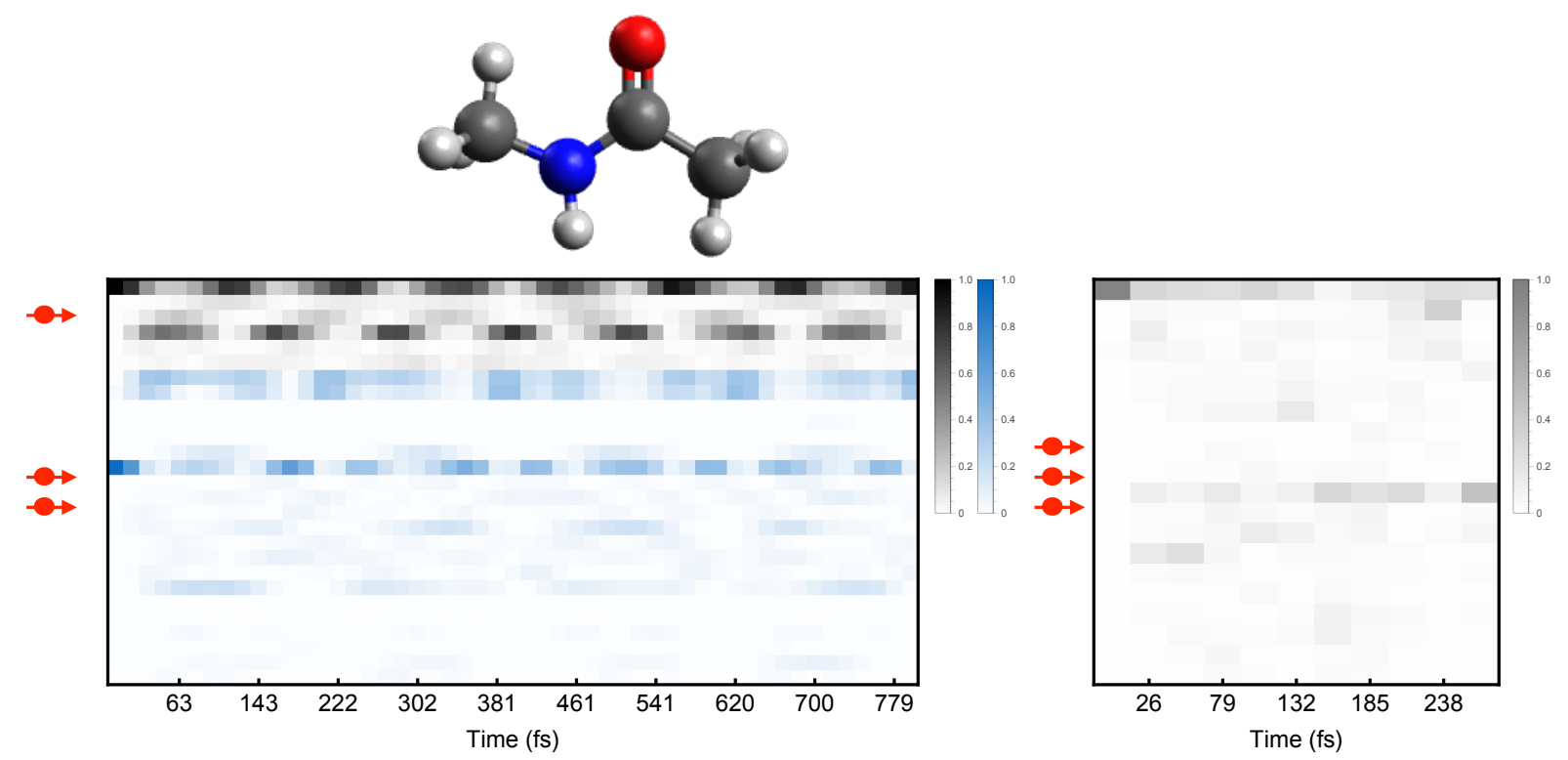

FIG. 8. NMA data. Full data sets for $|1\rangle$ (black), $|1\rangle|1\rangle$ (blue) and $|1\rangle|1\rangle|1\rangle$ (grey) input experiments.

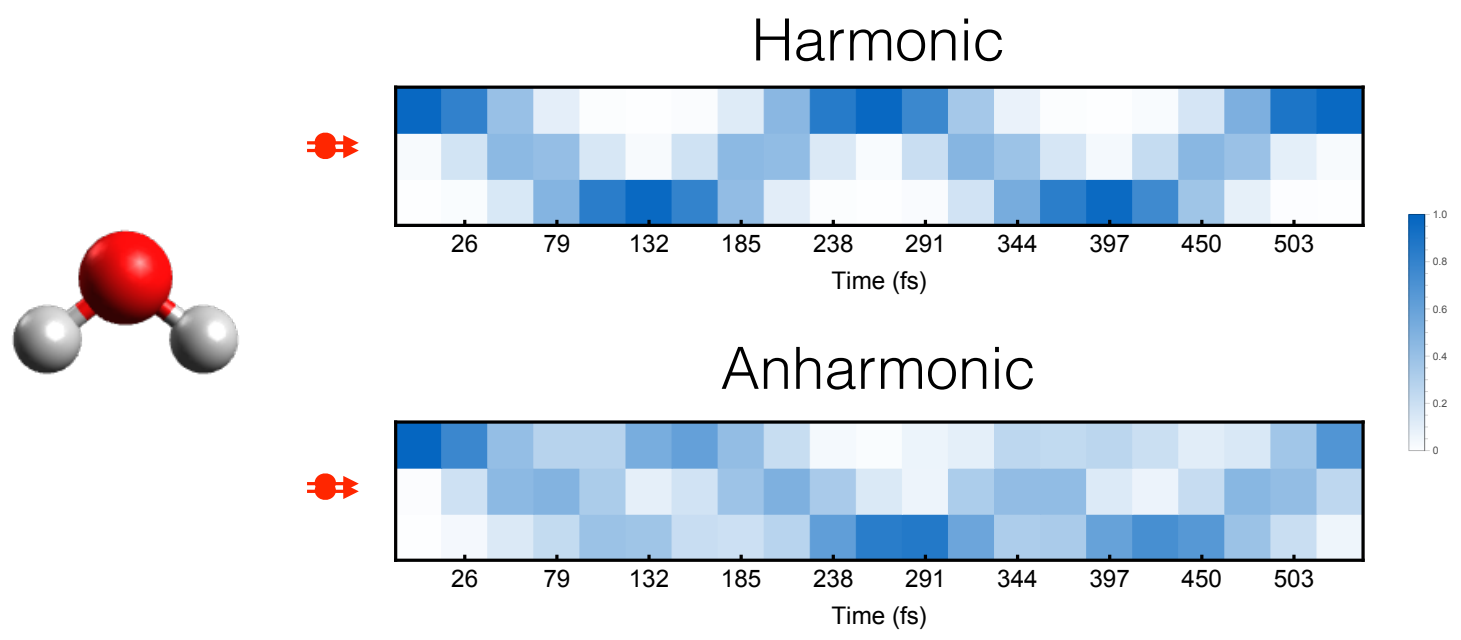

FIG. 9. $\mathrm{H}_{2} \mathrm{O}$ data. Full data sets for $|2\rangle$ and $|2\rangle|1\rangle$ input experiments for harmonic and anharmonic. States are ordered top to bottom as $\{|20\rangle,|11\rangle,|02\rangle\}$ 

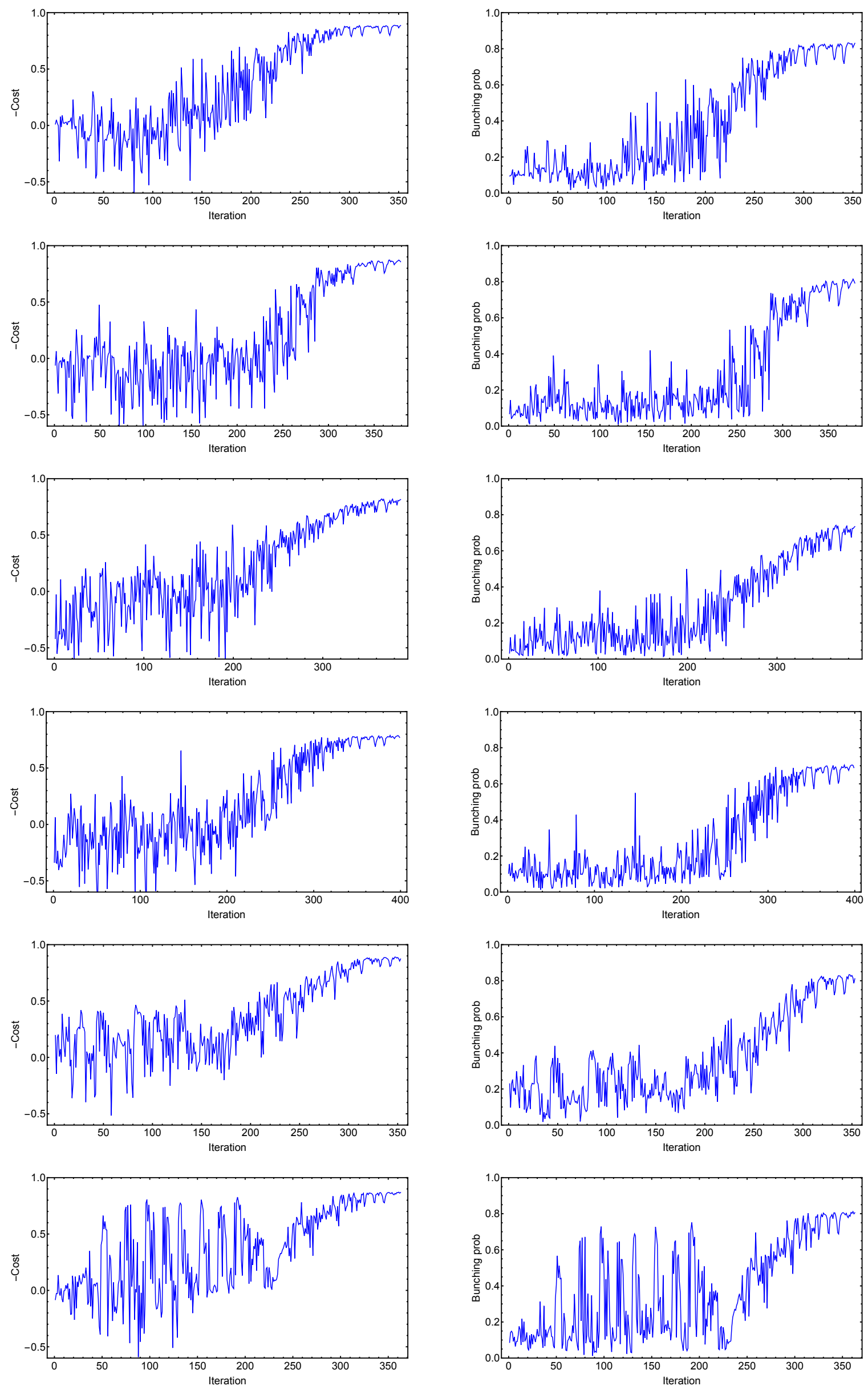

FIG. 10. Cost function (left) and probability of bunching in an NH stretch mode averaged over all timesteps (right) for a series of random initial states in $\mathrm{NH}_{3}$ dissociation experiment. 

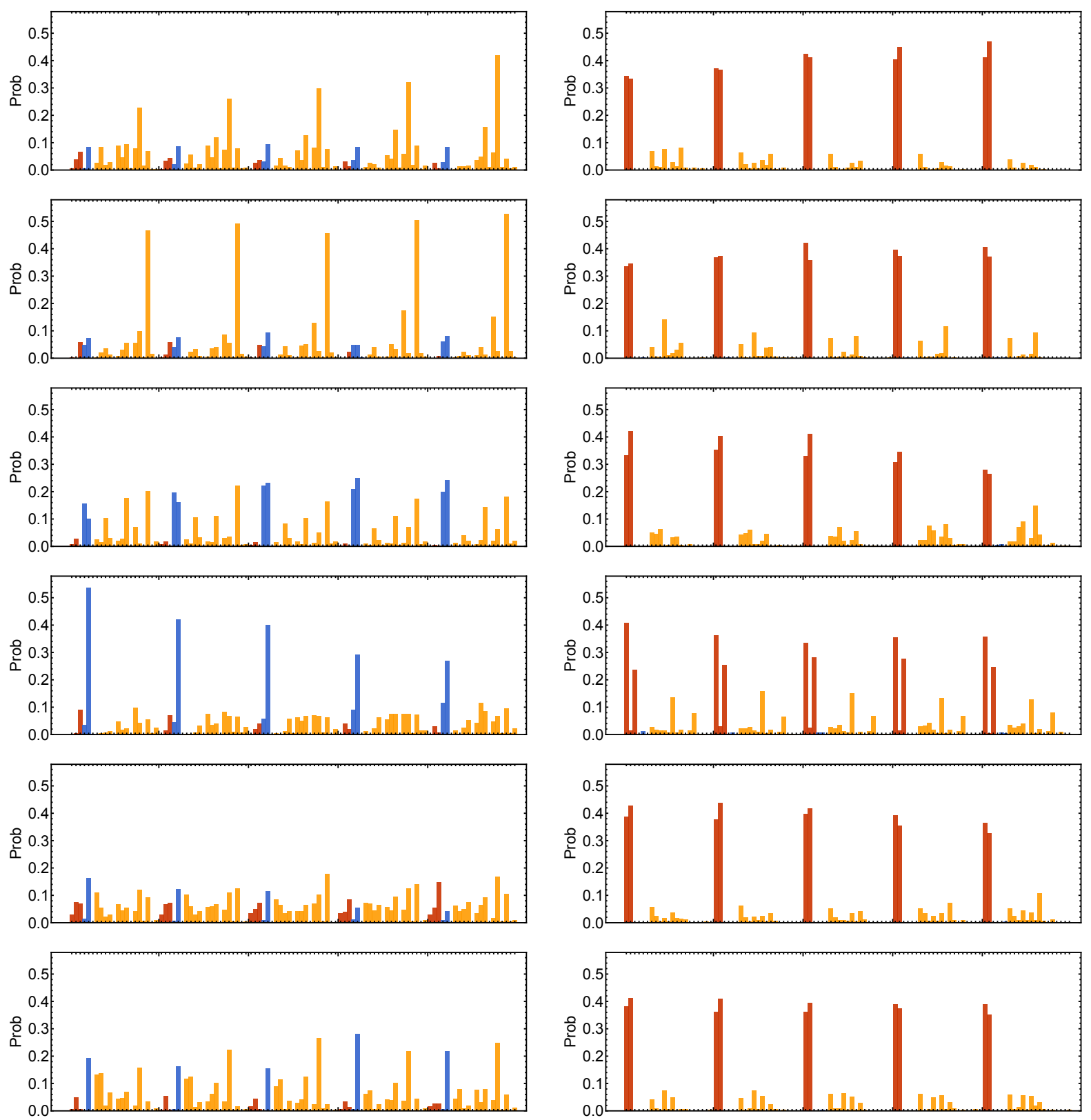

FIG. 11. Full distributions for initial (left column) and final (right column) states over the five time steps for the dissociation experiments summarised in Fig. 10. Red bars are probability of bunching in an NH stretch mode, blue bars are remaining bunched states and yellow are anti-bunched states. 